\title{
Generalized Motion by Mean Curvature as a Macroscopic Limit of Stochastic Ising Models with Long Range Interactions and Glauber Dynamics
}

\author{
Markos A. Katsoulakis ${ }^{1 \star}$, Panagiotis E. Souganidis ${ }^{2 \star \star}$ \\ ${ }^{1}$ Center for Mathematical Sciences, University of Wisconsin-Madison, Madison, WI 53706, USA. \\ Current address: Department of Mathematics, Michigan State University, E. Lansing, \\ MI 48824, USA \\ ${ }^{2}$ Department of Mathematics, University of Wisconsin-Madison, Madison, WI 53706, USA
}

Received: 22 November 1993/in revised form: 7 July 1994

\begin{abstract}
We study the macroscopic limit of an appropriately rescaled stochastic Ising model with long range interactions evolving with Glauber dynamics as well as the corresponding mean field equation, which is nonlinear and nonlocal. In the limit we obtain an interface evolving with normal velocity $\theta \kappa$, where $\kappa$ is the mean curvature and the transport coefficient $\theta$ is identified by an effective Green-Kubo type formula. The above assertions are valid for all positive times, the motion of the interface being interpreted in the viscosity sense after the onset of the geometric singularities.
\end{abstract}

\section{Introduction}

Stochastic Ising models with long range interactions were introduced by Kač, Uhlenbeck and Hemmer in [KUH] (see also Lebowitz and Penrose [LP]) to justify the validity of the Van der Waal's phase diagram, as the interaction range $\gamma^{-1}$ tends to infinity. For a very comprehensive description of the equilibrium theory of systems with long-range potentials we refer to the paper by Hemmer and Lebowitz [HL].

Stochastic Ising models with Kač potentials evolving in time with Glauber dynamics - each spin undergoes in a random way a finite number of flips - have a surprisingly rich structure and exhibit a great variety of physically interesting effects like spinodal decomposition, development of interfaces, etc. We refer to the papers by De Masi, Orlandi, Presutti and Triolo [DOPT 1,2,3] for a systematic study of some of these properties as well as to the ones by Comets [C], Comets and Eisele [CE] and Lebowitz, Orlandi and Presutti [LOP] for other non-equilibrium properties for systems with Kač potentials.

The mesoscopic limit of the ferromagnetic stochastic Ising model evolving with Glauber dynamics, i.e. the behavior of the model as $\gamma \rightarrow 0$ when space is rescaled by $\gamma$ and time is kept fixed, was studied by De Masi, Orlandi, Presutti and Triolo

\footnotetext{
$\star$ Supported by ONR

$\star \star$ Partially supported by NSF, ARO, ONR and the Alfred P. Sloan Foundation
} 
in [DOPT1], who obtained a mean field equation, which is a nonlocal, nonlinear evolution equation, for the limiting averaged magnetization. Since in the mesoscopic limit time is not rescaled, each individual spin in a time unit undergoes only a finite, random number of flips. The deterministic behavior described by the mean field equation is a mean field effect due to the scaling of the interaction: many spins (infinitely many in the limit $\gamma \rightarrow 0$ ) feel essentially the same potential and while each of them behaves randomly, their average evolves deterministically, due to a law of large numbers effect. The full interaction only arises at longer times, when each spin, after many flips, reaches a (local) equilibrium distribution.

Here we study the macroscopic limit, i.e. the behavior as $\gamma \rightarrow 0$ when both space and time are rescaled simultaneously, of the ferromagnetic stochastic Ising model with Kač potentials and Glauber dynamics as well as the long time behavior of the corresponding mean field equation and the propagation of the resulting interfaces.

Before we describe our results we need to remind the reader of some of the rather important progress, which has been made during the last few years, to describe geometric evolutions, i.e. motion of (generalized) hypersurfaces, past the first time at which singularities occur. It is well known that surfaces evolving with normal velocity depending on the curvature tensor, the direction and the position may start smooth and yet develop singularities in finite time. It is, however, important both from the mathematical point of view as well as for applications to find a way to interpret this evolution past singularities. One of the most successful ideas in this direction, known as the level set approach, is to represent the surface as a level set - for definiteness the zero level set - of the solution of a certain degenerate parabolic partial differential equation, known as the geometric pde associated with the motion. The mathematical theory of the level set approach was developed by Evans and Spruck in [ES] for motion by mean curvature and Chen, Giga and Goto in [CGG] for more general situations using the theory of viscosity solutions for second-order pde's. We refer to the User's Guide by Crandall, Ishii and Lions [CIL] for a detailed overview of theory of viscosity solutions as well as to the papers by Soner [So1], Barles, Soner and Souganidis [BSS] and Ishii and Souganidis [IS] and the references therein for alternative formulations, extensions, discussion, etc.

Our first result in this paper is about the asymptotic behavior of the mean field equation. We prove that in the asymptotic limit $\lambda \rightarrow 0$, when space and time are scaled by $\lambda^{-1}$ and $\lambda^{-2}$ respectively, the mean field equation yields an interface, which propagates with normal velocity equal to $\theta \kappa+A$, where $\kappa$ is the mean curvature, $A$ is some appropriate constant and $\theta>0$ is a transport coefficient, which is related to the mobility and the surface tension of the interface. This evolution is global in time; past the singularities it is interpreted in the viscosity sense. The novelty of the result, besides dealing with a highly nonlinear, nonlocal equation, is the identification of $\theta$, through a Green-Kubo type formula, which does not appear either at the microscopic level (i.e. the Ising model) or at the level of the mean field equation and it is actually related to an averaging effect, which is taking place during the limiting process.

Our second result states that in the asymptotic limit $\gamma \rightarrow 0$, when space and time are scaled by $\gamma^{-1} \lambda^{-1}(\gamma)$ and $\lambda^{-2}(\gamma)$ respectively, where $\gamma^{-\rho^{*}} \lambda(\gamma) \rightarrow \infty$ as $\gamma \rightarrow 0$ for some $\rho^{*}>0$, the average magnetization develops an interface which moves as the one for the mean field equation. We also prove that, with the same scaling, with large probability the spins align themselves to form clusters, which are separated by (random) interfaces close to the evolving macroscopic interface. 
In addition to being interesting (at least we hope so) from the point of view of both mathematics and statistical mechanics, our results may be thought of as providing a justification, from microscopic considerations, to phenomenological theories of phase-transitions like sharp-interface models derived by thermodynamic arguments (see Gurtin [G]) and sometimes by scaling of a Landau-Ginzburg model (see Allen and Cahn $[\mathrm{AC}]$ ). Finally, our results may also be thought of as providing a theoretical justification of the validity of some Monte Carlo-type methods, which have long been implemented in the physics literature, to approximate mean curvature evolution at any time. Notice that the stochastic spin dynamics are unaffected by the possible appearance of singularities in the flow.

A result similar to ours about the asymptotic behavior of the mean field equation was obtained, independently and at the same time, by Jerrard in $[\mathrm{J}]$ for a different nonlinear (but local) equation proposed by Penrose in [P], which is a local approximation of the mean field equation studied here. Our proof here applies to this equation as does the proof of $[\mathrm{J}]$ to ours. Macroscopic limits for stochastic Ising models with nearest neighbor interaction (in two space dimensions) have been studied by Spohn [Sp]. We also remark that the authors in [KS] studied the macroscopic behavior for all times of an Ising model with Glauber-Kawasaki dynamics, which involve a nearest neighbor interaction spin flip delocalized by a rapid stirring mechanism. Finally, the asymptotic behavior of the mean field equation and the macroscopic limit of the Ising model for the specific scaling $\lambda(\gamma)=\left(\log \gamma^{-1}\right)^{-1 / 2}$, under the assumption that the resulting interface is smooth, which, of course, is true only for small time in general, was also studied by De Masi, Orlandi, Presutti and Triolo in [DOPT1] and [DOPT3]. (See also Bonaventura [B] for a related result under the same smoothness assumption, for the macroscopic limit of the GlauberKawasaki model.)

The paper is organized as follows: In Sect. 2 we describe the Ising model and the Glauber dynamics and recall the result of [DOPT1] about the mesoscopic limit. Section 3 discusses the properties of the mean field equation and recalls the weak theory of propagating fronts. In Sect. 4 we state and discuss in detail our results, which we then prove in Sect. 5 (asymptotics of mean field equation) and 6 (macroscopic limit of the Ising model). In the last section we also state some consequences and generalizations of the results.

\section{Ising Models with Long Range Interactions and Glauber Dynamics}

Here we describe, in a rather brief way, general ferromagnetic Ising models, i.e. spin systems, with long range interactions, given by Kač potentials evolving according to Glauber dynamics. For a much more involved and considerably more enlightening discussion we refer, for example, to the papers by De Masi, Orlandi, Presutti and Triolo [DOPT1,2] and Comets [C] as well as the monograph by De Masi and Presutti [DP] and the references therein.

Ising models are spin systems flipping between \pm 1 at random on a lattice, which is chosen to be the $N$-dimensional hypercube $\mathbb{Z}^{N}$. A spin configuration $\sigma$ is an element of the state (configuration) space

$$
\Sigma=\{-1,1\}^{\mathbb{Z}^{N}}
$$

We write $\sigma=\left\{\sigma(x) \mid x \in \mathbb{Z}^{N}\right\}$ and call $\sigma(x)$ the spin at $x$. 
The dynamics of the model consist of a sequence of flips. If $\sigma$ is the configuration before a flip at $x$, then after the flip the configuration is

$$
\sigma^{x}(y)= \begin{cases}-\sigma(x) ;, & \text { if } y=x, \\ \sigma(y), & \text { if } y \neq x\end{cases}
$$

We assume that a flip occurs at $x$, when the configuration is $\sigma$, with a rate $c_{\gamma}(x, \sigma)$, given by:

$$
c_{\gamma}(x, \sigma)=\frac{e^{-\beta h_{i}(x) \sigma(x)}}{e^{-\beta h_{\gamma}(x)}+e^{\beta h_{\gamma}(x)}},
$$

where $\beta>0$ is the inverse temperature and

$$
h_{\gamma}(x)=h+\sum_{y \neq x} J_{\gamma}(x, y) \sigma(y)
$$

$h>0$ is the external magnetization field, $J_{\gamma}$ is the Kač potential

$$
J_{\gamma}(x, y)=\gamma^{N} J(\gamma|x-y|) \quad\left(x, y \in \mathbb{Z}^{N}\right)
$$

and $\gamma^{-1}>0$ is the interaction range.

The potential $J$ is assumed to satisfy:

$$
\left\{\begin{array}{l}
J: \mathbb{R}^{N} \rightarrow[0, \infty) \text { has compact support and is symmetric i.e. } \\
J(x)=J(|x|) \quad\left(x \in \mathbb{R}^{N}\right) .
\end{array}\right.
$$

The Glauber dynamics is a Markov jump process on $\Sigma$ with generator given by

$$
L_{\gamma} f(\sigma)=\sum_{x \in \mathbb{Z}^{N}} c_{\gamma}(x, \sigma)\left[f\left(\sigma^{x}\right)-f(\sigma)\right],
$$

acting on cylindrical functions $f$ on $\Sigma$. (See Liggett [L] for the precise definitions.) The solution of the equation

$$
\frac{d}{d t} f_{t}=L_{\gamma} f_{t}, \quad f_{0}=f
$$

is given by

$$
f_{t}(\sigma)=\sum_{\sigma^{\prime}} e^{L_{i} t}\left(\sigma, \sigma^{\prime}\right) f\left(\sigma^{\prime}\right),
$$

where $e^{L_{\gamma} t}\left(\sigma, \sigma^{\prime}\right)$ are the transition probabilities of the process.

The full-stochastic jump process $\sigma_{t}$ is constructed as follows: The initial configurations $\sigma^{0}$ are randomly distributed according to some measure $\mu^{\gamma}$ on $\Sigma$. Given a $\sigma^{0}, \sigma_{t}=\sigma^{0}$ for an exponentially distributed waiting time with rate $\sum_{y} c\left(y, \sigma^{0}\right), \sigma_{t}$ jumps to a new configuration $\sigma^{1}=\sigma^{x}$ with probability $c\left(x, \sigma^{0}\right) / \sum_{y} c\left(y, \sigma^{0}\right)$. Then $\sigma_{t}=\sigma^{1}$ for another exponentially distributed waiting time with rate $\sum_{y} c\left(y, \sigma^{1}\right)$, etc. Notice that, in view of the positivity of $J$, the probability of a spin flip at $x$ is higher when the spin at $x$ is different from that of most of its neighbors than it is when the spin agrees with most of its neighbors. Thus the system prefers configurations in which the spins tend to be aligned with one another. This property in the language of statistical mechanics, is referred to as ferromagnetism.

The particular choice of the Glauber dynamics is made in order to describe the transition (evolution as $t \rightarrow \infty$ ) of the initial measure $\mu^{\gamma}$ on $\Sigma$ towards the Gibbs 
measures (equilibrium measures) of the ferromagnetic Ising model. It turns out that in the presence of an external magnetization field, i.e. when $h \neq 0$, there is a unique Gibbs measure. The same is also true when $h=0$ but only for subcritical inverse temperatures $\beta \leqq \beta_{c}^{\gamma}$, where $\beta_{c}^{\gamma}$ is some constant determined by the specific model. On the other hand (see ([L]), when $\beta>\beta_{c}^{\gamma}$ there are more than one equilibrium measures, i.e. phase transitions take place.

A very basic question in the theory of stochastic Ising models with Kac potentials is the behavior of the system as the interaction range tends to infinity, i.e. $\gamma \rightarrow 0$. The passage in the limit $\gamma \rightarrow 0$ of quantities like the thermodynamical pressure, total magnetization, etc. is known as the Lebowitz-Penrose limit (see [LP, HL, DP], etc.).

Along these lines De Masi, Orlandi, Presutti and Triolo (see [DOPT1]) studied the asymptotics, as $\gamma \rightarrow 0$, of the averaged magnetization

$$
m_{\gamma}(x, t)=\mathbb{E}_{\mu ;}^{\gamma} \sigma_{t}(x) \quad\left((x, t) \in \mathbb{Z}^{N} \times[0, \infty)\right)
$$

of the system, where $\mathbb{E}_{\mu i}^{\gamma}$ denotes the expectation of the Glauber process starting from a measure $\mu^{\gamma}$, and obtained a mean field equation

$$
m_{t}+m-\tanh \beta(J * m+h)=0 \text { in } \mathbb{R}^{N} \times[0, \infty),
$$

where $J * m$ denotes the usual convolution in $\mathbb{R}^{N}$.

This equation is one of the points of interest of the paper. For completeness we state below the theorem of [DOPT1] which relates (2.2) and (2.3). To this end, we need to introduce for each $n \in \mathbb{Z}^{+}$the sets

$$
\mathbb{Z}_{n}^{N}=\left\{\underline{x}=\left(x_{1}, \ldots, x_{n}\right) \in\left(\mathbb{Z}^{N}\right)^{n} \mid x_{1} \neq \ldots \neq x_{n}\right\} .
$$

Theorem 2.1. ([DOPT1]). Assume that the Glauber process has as initial measure a product measure $\mu^{\gamma}$ such that

$$
\mathbb{E}_{\mu i}^{\gamma}(\sigma(x))=m_{0}(\gamma x) \quad\left(x \in \mathbb{Z}^{N}\right),
$$

where $m_{0}$ is Lipschitz continuous and (2.1) holds. Then, for each $n \in \mathbb{Z}^{+}$,

$$
\lim _{\gamma \rightarrow 0} \sup _{\underline{x} \in \mathbb{Z}_{n}^{N}}\left|\mathbb{E}_{\mu^{i}}\left(\prod^{n} \sigma_{t}\left(x_{l}\right)\right)-\prod_{l=1}^{n} m\left(\gamma x_{i}, t\right)\right|=0,
$$

where $m$ is the unique solution of (2.3) with initial datum $m_{0}$.

Theorem 2.1 was proved in [DOPT1] under the assumption that in addition to (2.1) $J$ is also in $C^{3}$. A more careful look at the proof reveals, however, that (2.1) suffices.

\section{The Mean Field Equation - Generalized Motion of Hypersurfaces}

(i) Properties of the mean field equation. We begin rewriting the mean field equation as

$$
m_{t}+m-\tanh \beta(J * m+\lambda a)=0 \quad \text { in } \quad \mathbb{R}^{N} \times[0, \infty),
$$


where $\lambda, a \in \mathbb{R}$. The reason for setting $h=\lambda a$ in (2.3) will become clear later in the paper. Equation (3.1) is, of course, nonlinear and nonlocal, due to the presence of the convolution. Nevertheless, since $J \geqq 0,(3.1)$ admits a comparison principle between solutions, i.e. if $w_{1}, w_{2}$ solve (3.1), then

$$
\text { if } w_{1} \leqq w_{2} \text { on } \mathbb{R}^{N} \times\{0\} \text {, then } w_{1} \leqq w_{2} \text { on } \mathbb{R}^{N} \times(0, \infty) \text {. }
$$

In this paper we will need a more detailed comparison, which we state later in this section.

The mean field equation admits three steady state solutions $m_{\beta, a \lambda}^{-}<m_{\beta, a \lambda}^{0}<$ $m_{\beta, a \hat{\imath}}^{+}$, i.e. solutions of the algebraic equation

$$
m=\tanh \beta(\hat{J} m+a \lambda)
$$

provided

$$
\beta \hat{J}>1
$$

where

$$
\hat{J}=\int_{\mathbb{R}^{N}} J(r) d r=\int_{\mathbb{R}} J(|r|)|r|^{N-1} d|r|,
$$

and

$$
|a \lambda| \leqq a_{0} \text { for some } a_{0}>0 \text {. }
$$

In the case $\lambda a=0$,

$$
m_{\beta, 0}^{ \pm}= \pm m_{\beta} \quad \text { and } \quad m_{\beta, 0}^{0}=0 .
$$

Observe that the steady state solutions are equilibria of the underlying ordinary differential equation, with $m_{\beta, a \lambda}^{ \pm}$being the stable and $m_{\beta, a \lambda}^{0}$ the unstable one.

An issue which we will study in this paper is whether solutions of (3.1) converge to the stable equilibria, as $t \rightarrow \infty$, and, if yes, to identify the regions in $\mathbb{R}^{N} \times$ $(0, \infty)$, where they converge to $m_{\beta, a \lambda}^{+}$and $m_{\beta, a \lambda}^{-}$.

A crucial tool towards studying this question is the existence of special solutions of (3.1), known as traveling waves, of the form

$$
m(r, t)=q\left(r-c^{\lambda}(a) t, a \lambda\right)
$$

which connect the stable equilibria, i.e.

$$
\dot{q}>0 \quad \text { in } \mathbb{R} \text { and } q( \pm \infty, a \lambda)=m_{\beta, a \lambda}^{ \pm} .
$$

The existence of such solutions is equivalent to the existence of a pair $\left(q, c^{\lambda}(a)\right)$ solving

$$
c^{\lambda}(a) \dot{q}(\xi, a \lambda)+q(\xi, a \lambda)=\tanh \beta[\tilde{J} * q(\xi, a \lambda)+a \lambda] \quad(\xi \in \mathbb{R}),
$$

where

$$
\tilde{J}(\zeta)=\int_{\mathbb{R}^{N-1}} J\left(\left(\zeta^{2}+\left|r^{\prime}\right|^{2}\right)^{1 / 2} d r^{\prime}\right.
$$


It turns out (see Theorem 2.4 of [DGP]) that there exist positive constants $a_{1}, a_{2}$ and $a_{3}$ such that the following hold uniformly for all $\lambda$ sufficiently small:

$$
\left\{\begin{array}{l}
\text { (i) } \lim _{\xi \rightarrow \pm \infty} e^{a_{1}|\xi|}\left|q(\xi, a \lambda)-\left[m_{\beta, a \lambda}^{ \pm} \pm \frac{a_{2}}{a_{1}} e^{-a_{1}|\xi|}\right]\right|=0 \\
\text { (ii) } \lim _{|\xi| \rightarrow \infty} e^{a_{1}|\xi|}\left|\dot{q}(\xi, a \lambda)-a_{2} e^{-a_{1}|\xi|}\right|=0 \\
\text { (iii) } \lim _{\xi \rightarrow \pm \infty} e^{a_{1}|\xi|} \mid \ddot{q}(\xi, a \lambda) \pm a_{1} a_{2} e^{-a_{2}|\xi|}=0 \\
\text { (iv) } a_{3} \geqq \frac{\tilde{J} * \dot{q}(\xi, a \lambda)}{\dot{q}(\xi, a \lambda)}>a_{3}^{-1} \\
\text { and } \\
\text { (v) } a_{3} \geqq \frac{\int J(|y|) \dot{q}(\xi+e \cdot y, a \lambda)(e \cdot y)^{2} d y}{\dot{q}(\xi, a \lambda)} \geqq a_{3}^{-1} \\
\text { for all unit vectors } e \in \mathbb{R}^{N}
\end{array}\right.
$$

and

$$
\left\{\begin{array}{l}
\text { (i) } c^{\lambda}(a) \rightarrow 0, q(\cdot, \operatorname{ta} \lambda) \rightarrow q(\cdot, 0) \text { and } \dot{q}(\cdot, a \lambda) \rightarrow \dot{q}(\cdot, 0) \text { as } \lambda \rightarrow 0 \\
\text { the last two limits being uniform in } \mathbb{R}, \\
\text { (ii) } \lambda^{-1} c^{\lambda}(a) \rightarrow c(a) \text { as } \lambda \rightarrow 0 \\
\text { and } \\
\text { (iii) } c(a) \rightarrow 0 \text { as } a \rightarrow 0 .
\end{array}\right.
$$

We conclude our discussion about the basic properties of the mean field equation with a lemma about a strict local comparison between solutions of equations like (3.1). Since its proof is a straightforward modification of an analogous result in [DOPT2] we omit it.

Lemma 3.1. Let the bounded functions $\underline{m}$ and $\bar{m}$ satisfy

$$
\bar{m}_{t}+\bar{m}-\tanh \beta[J * \bar{m}+\bar{h}] \geqq 0 \quad \text { in } \quad \mathbb{R}^{N} \times[a, b]
$$

and

$$
\underline{m}_{t}+\underline{m}-\tanh \beta[J * \underline{m}-\underline{h}] \leqq 0 \text { in } \mathbb{R}^{N} \times[a, b],
$$

where $\underline{h}, \bar{h}, b-a \in[0,1]$. There exists $C=C\left(\|\bar{m}\|_{\infty},\|\underline{m}\|_{\infty}\right)<\beta$ such that for all $L>0$ and all $(r, t) \in B(0, L) \times[a, b]$,

$$
\begin{aligned}
\underline{m}(r, t) & -\bar{m}(r, t) \leqq\|J\|_{\infty}^{1 / 2}|\operatorname{spt}(J)| e^{(1+\beta)(t-a)}\left[\sup _{|r| \leqq L}\left(\underline{m}\left(r^{\prime}, a\right)-\bar{m}\left(r^{\prime}, a\right)\right)^{+}\right. \\
& \left.+(L-|r|)^{-1 / 2} e^{(L-|r|)[1-\log (L-|r|)]}[\beta(t-a)]^{L-|r|}\right]-\beta(\bar{h}+\underline{h}) C(t-a) .
\end{aligned}
$$

(ii) Weak front propagation. Next we recall the definition of generalized motions of surfaces with normal velocity

$$
V=\theta \kappa+A,
$$

where $\theta>0, A \in \mathbb{R}$ and $\kappa$ is the mean curvature. 
As mentioned in the Introduction such evolutions can start out smooth and yet develop singularities at a later time. A great deal of work has been done over the last few years in order to interpret the evolution past singularities, the main idea being representing the surface as a level set, for definiteness the zero level set, of the solution of a certain degenerate parabolic partial differential equation. The mathematical theory of the level set approach was developed by Evans and Spruck in [ESp] for motion by mean curvature and Chen, Giga and Goto in [CGG] for more general situations using the theory of viscosity solutions. We refer to the User's Guide by Crandall, Ishii and Lions ([CIL]) for a detailed overview of the theory of viscosity solutions as well as to the papers by Soner [S], Barles, Soner and Souganidis [BSS], Ishii and Souganidis [IS], Katsoulakis and Souganidis [KS], etc. for alternative formulations, extensions, discussion, etc.

Let $\Omega_{0} \subset \mathbb{R}^{N}$ be open and

$$
\Gamma_{0}=\mathbb{R}^{N} \backslash\left(\Omega_{0} \cup \bar{\Omega}_{0}^{c}\right) .
$$

The generalized evolution $\Gamma_{t}$ of $\Gamma_{0}$ is defined as

$$
\Gamma_{t}=\left\{r \in \mathbb{R}^{N}: w(r, t)=0\right\},
$$

where $w$ is the unique viscosity solution of

$$
\left\{\begin{array}{l}
w_{t}-\theta \operatorname{tr}\left(I-\frac{D w \otimes D w}{|D w|^{2}}\right) D^{2} w-A|D w|=0 \text { in } \mathbb{R}^{N} \times(0, \infty), \\
w=d_{0} \text { on } \mathbb{R}^{N} \times\{0\},
\end{array}\right.
$$

and

$$
d_{0}(r)=\left\{\begin{array}{ll}
\operatorname{dist}\left(r, \Gamma_{0}\right) & \text { if } r \in \Omega_{0}, \\
-\operatorname{dist}\left(r, \Gamma_{0}\right) & \text { if } r \in \bar{\Omega}_{0}^{c}
\end{array} .\right.
$$

For the details and justification of the above definition we refer to the references mentioned above.

\section{The Main Results}

(i) Asymptotics of the mean field equation. The first result of the paper is about the asymptotics, in the limit $\lambda \rightarrow 0^{+}$, of the following rescaled version of (3.1):

$$
m_{t}^{\hat{\lambda}}+\lambda^{-2}\left[m^{\lambda}-\tanh \beta\left(J^{\lambda} * m^{\lambda}+\lambda a\right)\right]=0 \quad \text { in } \quad \mathbb{R}^{N} \times(0, \infty),
$$

where

$$
J^{\lambda}(r)=\lambda^{-N} J\left(\lambda^{-1} r\right) \quad\left(r \in \mathbb{R}^{N}\right) .
$$

Equation (4.1) follows from (3.1) by writing

$$
m^{\lambda}(r, t)=m\left(\lambda^{-1} r, \lambda^{-2} t\right) \quad\left((r, t) \in \mathbb{R}^{N} \times(0, \infty)\right) .
$$

In the limit $\lambda \rightarrow 0$ and for supercritical inverse temperatures $\beta$, i.e. when (3.4) holds, we show that there exists a transport coefficient

$$
\theta=\frac{\beta}{2} \iint J(|y|) \dot{q}(\xi, 0) \dot{q}(\xi+e \cdot y, 0)(\hat{e} \cdot y)^{2} d y d \xi / \int \frac{(\dot{q}(\xi, 0))^{2}}{1-q^{2}(\xi, 0)} d \xi,
$$


where $e, \hat{e}$ are two orthogonal unit vectors in $\mathbb{R}^{N}$, such that the solution $m^{\hat{\lambda}}$ of (4.1) converges at each point of $\mathbb{R}^{N} \times(0, \infty) \backslash \bigcup_{t>0} \Gamma_{t} \times\{t\}$ to the stable steady solutions $m_{\beta, 0}^{ \pm}$of (4.1). The surface $\Gamma_{t}$, which is known as the antiphase boundary, is moving with normal velocity

$$
V=\theta \kappa+c(a)
$$

where $c(a)$ is given by (3.13). As a matter of fact we show that $m^{\lambda} \rightarrow m_{\beta, 0}^{+}$inside the front and $m^{\lambda} \rightarrow m_{\beta, 0}^{-}$outside. Note that due to the symmetry of $J, \theta$ in (4.2) is independent of the particular choice of $e$ and $\hat{e}$. Finally, all the above will be proved under the assumption the system begins at a local equilibrium, i.e. that

$$
m^{\lambda}=q\left(\frac{d_{0}}{\lambda}, a \lambda\right) \quad \text { on } \quad \mathbb{R}^{N} \times\{0\},
$$

where $d_{0}$ is the signed distance function from a closed set $\Gamma_{0} \subset \mathbb{R}^{N}$ and $q$ is the traveling wave associated to $(4.1)$ such $q(0, a \lambda)=0$. We will discuss in a future work how to eliminate this restriction. The precise result is:

Theorem 4.1. Assume (2.1) and (3.4) and let $m^{\lambda}$ be the solution of (4.1) with initial datum (4.3), where $\Gamma_{0}=\partial \Omega_{0}, \Omega_{0} \subset \mathbb{R}^{N}$ being open. Then, as $\lambda \rightarrow 0^{+}$,

$$
m^{\lambda} \rightarrow\left\{\begin{array}{lc}
m_{\beta, 0}^{+} & \{w>0\}, \\
m_{\beta, 0}^{-} & \text {locally uniformly in } \\
& \{w<0\},
\end{array}\right.
$$

where $m_{\beta, 0}^{ \pm}$are given by (3.3), $w$ is the unique solution of (3.17) with initial datum $d_{0}, \theta$ is given by (4.2) and $A=c(a)$, the latter given by (3.13).

As mentioned in the Introduction, under the additional assumption that $\Gamma_{0}$ is smooth and only for the time interval during which the evolution $\left(\Gamma_{t}\right)_{t \geq 0}$ remains smooth, Theorem 4.1 was proved (for $a=0$ ) by De Masi, Orlandi, Presutti and Triolo in [DOPT3].

A result analogous to Theorem 4.1 but for a local approximation of (4.1) in [P] was proved independently by Jerrard (see $[\mathrm{J}]$ ). We remark that results analogous to Theorem 4.1 but for the reaction-diffusion equation were first obtained in this generality by Evans, Soner and Souganidis in [ESS] (see also [BSS]). We refer to these works for the history of the problem as well as more recent papers.

Notice that Theorem 4.1 yields no information for the limit of $m^{\lambda}$ on the front, which may have, in principle positive $N$-dimensional Lebesgue measure, i.e. fattening may occur. For general conditions for non-fattening we refer to [BSS]. In the case of the reaction-diffusion equation, information about the limit where fattening occurs was obtained by Ilmanen in [I] (see also Soner [So2]). We hope to return to this issue for the problem at hand in a future paper.

We conclude the first part of this section with a brief discussion about the ideas involved in proving Theorem 4.1. The proof is based partially on refinements of a number of ideas introduced by Barles, Soner and Souganidis in [BSS] to study asymptotic limits of general reaction-diffusion equations. The approach of [BSS] is different from the one introduced by Evans, Soner and Souganidis in [ESS] to study the asymptotics of the Allen-Cahn equation. The difference is that [ESS] constructs super- and sub-solutions of the reaction-diffusion equations. On the contrary [BSS] 
studies the problem at the level $\lambda=0$ using a number of sophisticated and new techniques to compensate for the lack of estimates. There is, however, a serious difficulty to apply directly [BSS] to (4.1). This is due mainly to the highly nonlinear form of the equation and to some extent its nonlocal character and is related to the identification of the transport coefficient $\theta$ in (4.3). This coefficient comes up because of an averaging effect occurring in the limit $\lambda \downarrow 0$ of the jump process, corresponding to the linearization of (4.1) around $q$. To study this averaging from the analytical point of view, we need to employ ideas from the theory of homogenization for viscosity solutions, which were introduced by Lions, Papanicolaou and Varadhan [LPV] and further developed by Evans [E]. The combination of analytic techniques from both propagation of fronts and homogenization is nontrivial. Our paper as well as Jerrard's ([J]) are the first ones in this direction.

(ii) Macroscopic limits of the ferromagnetic Ising model. The second major result of the paper is about the existence of a macroscopic scaling of the form

$$
(x, t) \rightarrow\left(\gamma \lambda(\gamma) x, \lambda^{2}(\gamma) t\right) \quad\left(x \in \mathbb{Z}^{N}\right),
$$

where $\lambda(\gamma)$ is to be determined, so that in the limit the appropriately rescaled averaged magnetization yields an interface moving according to a macroscopic equation of the type given by (4.3). As a matter of fact one would like to obtain, and we do so below, a propagation of chaos type result at this scaling. Such a result, of course, should be global in time, i.e. to hold past the geometric singularities, since the spins, by definition, are not sensitive to the regularity of the macroscopic profile.

As in the case of the asymptotics of the mean field equation, here we will assume that our initial measure is at a local equilibrium. More precisely, we assume:

$$
\left\{\begin{array}{l}
\text { (i) } \mu^{\gamma} \text { is a product measure on } \Sigma \\
\text { and } \\
\text { (ii) } E_{\mu \nu^{\prime}}^{\gamma} \sigma(x)=q\left(\frac{d_{0}(\gamma \lambda(\gamma) x)}{\lambda(\gamma)}, 0\right) \quad\left(x \in \mathbb{Z}^{N}\right),
\end{array}\right.
$$

where $q$ is the traveling wave corresponding to (3.1) with $a=0$ and $q(0,0)=0$, $d_{0}$ is the signed distance function from a closed set $\Gamma_{0} \subset \mathbb{R}^{N}$ and $\lambda(\gamma)$ will be specified below.

To state our result we need to introduce some notation and special sets. To this end, for $\gamma>0$, and $\rho_{1}>0$ set

$$
Q_{\gamma}=\gamma^{-1}\left[-\gamma^{-\rho_{1}}, \gamma^{-\rho_{1}}\right]^{N} \cap \mathbb{Z}^{N},
$$

where $\rho_{1}$ is to be chosen and $[-R, R]^{N}$ denotes the hypercube of side $R$ in $\mathbb{R}^{N}$.

Let $w$ be the solution of the geometric pde

$$
\left\{\begin{array}{l}
w_{t}-\theta \operatorname{tr}\left(I-\frac{D w \otimes D w}{|D w|^{2}}\right) D^{2} w=0 \text { in } \mathbb{R}^{N} \times(0, \infty), \\
w=d_{0} \text { on } \mathbb{R}^{N} \times\{0\}
\end{array}\right.
$$

and define, for $t>0$, the sets

$$
\left\{\begin{array}{l}
P_{t}^{\gamma}=\left\{x \in \mathbb{Z}^{N}: w(\gamma \lambda(\gamma) x, t)>0\right\} \\
\text { and } \\
N_{t}^{\gamma}=\left\{x \in \mathbb{Z}^{N}: w(\gamma \lambda(\gamma) x, t)<0\right\}
\end{array}\right.
$$


and

$$
M_{\gamma, t}^{n}=\left\{\underline{x} \in \mathbb{Z}_{n}^{N}: x_{i} \in Q_{\gamma} \cap\left(P_{t}^{\gamma} \cup N_{t}^{\gamma}\right)\right\} \quad(t>0),
$$

where $\mathbb{Z}_{n}^{N}$ is given by (2.3).

Our result is:

Theorem 4.2. Assume (2.1) and (3.4). There exists a $\rho^{*}>0$, given by Lemma 6.4, such that for any $\lambda(\gamma)$ such that $\gamma^{-\rho^{*}} \lambda(\gamma) \rightarrow+\infty$ as $\gamma \rightarrow 0$, if (4.5) holds then, for all $t \in(0, \infty)$,

$$
\lim _{\gamma \rightarrow 0} \sup _{\underline{x} \in M_{i, t}^{n}}\left|E_{\mu^{i}}^{\gamma} \prod_{i=1}^{n} \sigma_{t \lambda(\gamma)^{-2}}(x)-m_{\beta}^{n} \prod_{x_{l} \in N_{t}^{\gamma}}(-1)\right|=O(\lambda)
$$

with the limit local uniform in $t$, where $N_{t}^{\gamma}$ and $M_{\gamma, t}^{n}$ are given by (4.8) and (4.9).

Before we continue we remark that the critical $\rho^{*}$ in Theorem 4.2 is independent of the particular value of the inverse temperature $\beta$. Finally, notice, as in Theorem 4.1, Theorem 4.2 yields no information when fattening occurs. We hope to return to this issue in a future paper.

Theorem 4.2 is the first global result showing the convergence (macroscopic limit) of the scaled averaged magnetization of the ferromagnetic Ising model with $\mathrm{Kac}$ potential to the mean curvature flow with the appropriate transport coefficient past the first time geometric singularities occur. A short time result, i.e. the convergence as long as the geometric flow is smooth, was proved in [DOPT1]. An analogous result but for nearest neighbor interaction Ising models in two dimensions (where the curvature motion is always smooth) was obtained by Spohn in [Sp]. When the dynamics of nearest neighbor model are coupled with Kawasaki dynamics at infinite temperature, which tend to delocalize the interactions, the macroscopic limit was studied by Bonaventura (see [B]) for smooth motions (i.e. for small time) and by the authors (see $[\mathrm{KS}]$ ) in the generality of Theorem 4.2 and even when fattening occurs.

We conclude with a brief discussion of the proof of Theorem 4.2. We show, see Sect. 6 below, that the spin dynamics are close with respect to some seminorm and for short times to the solution of a discrete version of the mean field equation. On the other hand, this solution is close (in the $L^{\infty}$-norm and for short times) to the solution of the mean field equation - all the above are made precise in Sect. 6. In summary, we show that the spin dynamics are equal, in a heuristic sense, to the solution of the mean field equation for short times plus an error. This suggests that we should discretize in time (as it was done before in, among others, [DP, B, KS, DOPT1], etc.); the errors, however, add up! To overcome this difficulty, we introduce mean field equations with $a \neq 0$, i.e. we speed up (or down) the geometric motion or in the language of statistical physics we introduce a small external magnetization field. Using the sharp comparison principle stated in Sect. 3 we are then able to absorb the error at each time step and to conclude using Theorem 4.1.

\section{Proof of Theorem 4.1}

Here we present the proof of Theorem 4.1. Since it is rather long, we are going to split it into a number of propositions and lemmas with the hope that in this way 
it will become more transparent. Throughout this section we will be using $x, y$ etc. to denote points in $\mathbb{R}^{N}$. Since there will be no mentioning of the particle systems here, this will not create (we hope) any confusion.

Proof of Theorem 4.1. 1. The comparison principle for Eq. (4.1) and assumption (4.4) yield that

$$
m^{\lambda} \in\left(m_{\beta, a \lambda}^{-}, m_{\beta, a \lambda}^{+}\right) \text {in } \quad \mathbb{R}^{N} \times(0, \infty)
$$

hence we can introduce the change of variables

$$
m^{\lambda}=q\left(\frac{z^{\lambda}}{\lambda}, a \lambda\right) \quad \text { on } \quad \mathbb{R}^{N} \times[0, \infty) .
$$

2. Next we define the upper- and lower-semicontinuous functions

$$
z^{*}(x, t)=\varlimsup_{\substack{(y, s) \rightarrow(r, t) \\, \rightarrow 0}} z^{\lambda}(y, s) \text { and } z_{*}(x, t)={\underset{\substack{(y, s) \rightarrow(x, t) \\, \rightarrow 0}}{\lim }} z^{\lambda}(y, s),
$$

as well as their regularizations (in $x$ )

$$
\left\{\begin{array}{l}
\bar{z}(x, t)=\sup _{y \in \mathbb{R}^{N}}\left\{z^{*}(y, t)-|x-y|\right\} \\
\text { and } \\
\underline{z}(x, t)=\inf _{y \in \mathbb{R}^{N}}\left\{z_{*}(y, t)+|x-y|\right\} .
\end{array}\right.
$$

3. Let $t_{*}$ be the extinction time of the front $\left(\Gamma_{t}\right)_{t \geqq 0}$ evolving according to (4.3), i.e.

$$
t_{*}=\sup \left\{T:\left\{x \in \mathbb{R}^{N}: w(x, t)>0\right\} \neq \emptyset \text { for } t \in[0, T)\right\},
$$

where $w$ is as in the statement of the theorem.

Below we use the following proposition. Since its proof is rather long and complicated, we postpone it until after the proof of the theorem.

Proposition 5.1. Under the assumptions of Theorem 4.1, the function $\bar{z} \wedge 0$ (resp. $\underline{z} \vee 0)$ is a sub-(resp. super-) solution of the geometric pde (3.17) with $A=c(a)$ in $\mathbb{R}^{N} \times\left(0, t_{*}\right]$. Moreover, $\bar{z} \wedge 0=d_{0} \wedge 0$ and $\underline{z} \vee 0=d_{0} \vee 0$ on $\mathbb{R}^{N} \times\{0\}$. Hence, by the standard comparison principle for viscosity solutions,

$$
\bar{z} \wedge 0 \leqq w \wedge 0 \leqq w \wedge 0 \leqq \underline{z} \vee 0 \quad \text { on } \quad \mathbb{R}^{N} \times\left[0, t_{*}\right]
$$

4. The conclusion of Theorem 4.1 follows now immediately in $\mathbb{R}^{N} \times\left(0, t_{*}\right]$. Indeed, (5.3), (5.4) and (5.5) yield

$$
z^{*} \leqq w \text { in }\{w<0\} \text { and } z_{*} \geqq w \text { in }\{w>0\} .
$$

We can now conclude using (5.1), (5.2) and the fact that

$$
\varlimsup_{\lambda \rightarrow 0} q\left(\frac{\xi}{\lambda}, \lambda a\right)=-m_{\beta} \quad \text { if } \quad \xi<0 \quad \text { and } \quad \varliminf_{\lambda \rightarrow 0} q\left(\frac{\xi}{\lambda}, \lambda a\right)=m_{\beta} \text { if } \xi>0 \text {. }
$$


5. To go beyond the extinction time $t_{*}$, we argue exactly as in [ESS], observing that

$$
\{(x, t): w(x, t)<0\}=\bigcup_{\delta>0}\left\{(x, t): w^{\delta}(x, t)<0\right\},
$$

where, for each $\delta>0, w^{\delta}$ is the unique solution of (3.17) with $A=c(a)$ and initial datum $d_{0}+\delta$. This together with the fact that distance between two surfaces evolving according to (4.3) increases in time, concludes the proof.

We continue preparing the ground towards the proof of Proposition 5.1, which is, of course, the heart of our argument and which will follow from the two lemmas we state below.

To this end, if

$$
T=\sup \left\{T^{\prime}:\left|z^{*}\right|,\left|z_{*}\right|<\infty \quad \text { in } \quad \mathbb{R}^{N} \times\left[0, T^{\prime}\right)\right\},
$$

then we have:

Lemma 5.2. Under the assumptions of Theorem 4.1, $T$ is positive.

Lemma 5.3. Under the assumptions of Theorem 4.1, the conclusions of Proposition 5.1 hold in $\mathbb{R}^{N} \times[0, T]$.

We postpone the proofs of Lemmas 5.2 and 5.3 for later in this section and we continue with the proof of Proposition 5.1.

Proof of Proposition 5.1. 1. If $\bar{z} \wedge 0$ (resp. $\underline{z} \vee 0$ ) is a sub- (resp. super-) solution of (3.17) in $\mathbb{R}^{N} \times[0, T)$, then $\bar{z} \wedge 0$ is a sub-(resp. super-) solution of (3.17) in $\mathbb{R}^{N} \times[0, T]$.

This is a standard observation in the theory of parabolic equations, provided $\bar{z} \wedge 0$ and $\underline{z} \vee 0$ are bounded. In our context we can always reduce to this situation by considering the functions $\phi(\bar{z} \wedge 0)$ and $\phi(\underline{z} \vee 0)$, where $\phi: \mathbb{R} \rightarrow \mathbb{R}$ is strictly increasing and continuous and using the fact that the scaling properties of (3.17) imply that $\phi(\underline{z} \wedge 0)$ is also a sub- (resp. super-) solution. We conclude by applying $\phi^{-1}$.

2. In view of Lemmas 5.2 and 5.3 and the above observation, it suffices to show

$$
T \geqq t_{*},
$$

recall $t_{*}$ is the extinction time of the front $\left(\Gamma_{t}\right)_{t \geqq 0}$.

3. Suppose that

$$
T<t_{*} .
$$

Then Lemma 5.3 and the observation above yield that

$$
\left\{\begin{array}{l}
\bar{z} \wedge 0 \leqq w \wedge 0 \text { and } \underline{z} \vee 0 \geqq w \vee 0 \text { on } \mathbb{R}^{N} \times\{T\} \\
\text { and } \\
\left\{x \in \mathbb{R}^{N}: w(x, T)<0\right\} \neq \emptyset \text { and }\left\{x \in \mathbb{R}^{N}: w(x, T)>0\right\} \neq \emptyset,
\end{array}\right.
$$

i.e. there exist $\bar{x}, \underline{x} \in \mathbb{R}^{N}$ such that

$$
\bar{z}(\bar{x}, T)<0 \text { and } \underline{z}(\underline{x}, T)>0 .
$$

Since $\bar{z}$ and $\underline{z}$ are respectively upper- and lower-semicontinuous functions, there exists an $\varepsilon_{0}>0$ such that

$$
\bar{z}(\bar{x}, T+\varepsilon)<0 \text { and } \underline{z}(\underline{x}, T+\varepsilon)>0 \text { for } \varepsilon \leqq \varepsilon_{0} .
$$


But then the definitions of $\bar{z}, \underline{z}, z^{*}$ and $z_{*}$ yield, for all $y \in \mathbb{R}^{N}$ and $\varepsilon \in\left(0, \varepsilon_{0}\right]$,

$$
-|\bar{x}-y| \leqq z_{*}(y, T+\varepsilon) \leqq z^{*}(y, T+\varepsilon) \leqq|\bar{x}-y|
$$

which, of course, contradicts the definition of $T$.

To prove Lemmas 5.2 and 5.3 we will need a technical lemma, which we state and prove below.

Lemma 5.4. Assume that $J$ satisfies (2.1) and let $\xi \rightarrow q(\xi, a \lambda)$ be the unique traveling wave solution of (3.1) with $q(0, a \lambda)=0$. If $e$ is a unit vector in $\mathbb{R}^{N}$ and $\xi, \tau \in \mathbb{R}$ are such that $\operatorname{sgn}(\xi)(\tau-1) \leqq 0$, then

$$
\int J(|y|) q(\xi+\tau e \cdot y, a \lambda) d y \geqq \int J(|y|) q(\xi+e \cdot y, a \lambda) d y .
$$

Proof. 1. In view of the symmetry of $J$, it suffices to assume that $e=(1,0, \ldots, 0)$, in which case $(5.8)$ reduces to

$$
f(\tau)=\int J(|y|) q\left(\xi+\tau y_{1}\right) d y \geqq \int J(|y|) q\left(\xi+y_{1}\right) d y=f(1),
$$

where to simplify the notation we dropped the dependence of $q$ on $a \lambda$.

2. To conclude it suffices to show

$$
\operatorname{sgn}(\xi) f^{\prime}(\tau) \leqq 0 .
$$

To this end, assume $\xi>0$ (the argument for $\xi<0$ is similar) and compute

$$
\begin{aligned}
f^{\prime}(\tau)= & \int J(|y|) \dot{q}\left(\xi+\tau y_{1}\right) y_{1} d y=\int_{\left\{y_{1} \geqq 0\right\}} J(|y|)\left[\dot{q}\left(\xi+\tau y_{1}\right)-\dot{q}\left(\xi-\tau y_{1}\right) y_{1}\right] d y \\
= & \int_{\left\{0 \leqq y_{1} \leqq \frac{2 \xi}{\tau}\right\}} J(|y|)\left[\dot{q}\left(\xi+\tau y_{1}\right)-\dot{q}\left(\xi-\tau y_{1}\right)\right] y_{1} d y \\
& +\int_{\left\{y_{1}>\frac{2 \xi}{\tau}\right\}} J(|y|)\left[\dot{q}\left(\xi+\tau y_{1}\right)-\dot{q}\left(\xi-\tau y_{1}\right)\right] y_{1} d y .
\end{aligned}
$$

For $y_{1} \in[0,2 \xi / \tau]$ we have $-\xi \leqq \xi-\tau y_{1}$, so $\dot{q}\left(\xi-\tau y_{1}\right) \geqq \dot{q}\left(\xi+\tau y_{1}\right)$ and on $\left[2 \xi / \tau_{1},+\infty\right), \quad \xi-\tau y_{1}<-\xi<0$, thus $\dot{q}\left(\xi+\tau y_{1}\right)=\dot{q}\left(-\xi-\tau y_{1}\right) \leqq \dot{q}\left(\xi-\tau y_{1}\right)$. Hence $f^{\prime}(\tau) \leqq 0$.

Next we prove Lemma 5.2.

Proof of Lemma 5.2. 1. Let $\underline{M}_{0}: \mathbb{R}^{N} \rightarrow \mathbb{R}$ be a smooth approximation of $d_{0}$ from below such that, for some $\delta>0$,

$$
\operatorname{sign}\left(\underline{M}_{0}\right)\left(\left|D \underline{M}_{0}\right|-1\right) \leqq 0 \text { on } \mathbb{R}^{N} \text { and }\left|D \underline{M}_{0}\right|=1 \text { if } 0 \leqq \underline{M}_{0} \leqq \delta .
$$

2. Define the function

$$
\underline{M}^{\lambda}(x, t)=q\left(\frac{\underline{M}_{0}(x)-\underline{\alpha} t}{\lambda}, \lambda a\right),
$$

where $\underline{\alpha}>0$ is to be chosen below and $t \in[0, \underline{t}]$, where $\underline{t}>0$ is so small that $\underline{\alpha} t<\delta$ for $t \in[0, t]$. 
3. We claim that $\underline{M}^{\lambda}$ is a subsolution of (4.1) for $\lambda \leqq \lambda_{0}(\underline{\alpha}, \delta)$. Indeed insert $\underline{M}^{\lambda}$ in (4.1) and compute - to simplify the notation we drop the dependence of $q$ on $a \lambda$-recalling that $q$ satisfies $(3.10)$ :

$$
\begin{aligned}
\mathscr{L}\left(\underline{M}^{\hat{\lambda}}\right) & =\underline{M}_{t}^{\lambda}+\lambda^{-2}\left\{\underline{M}^{\lambda}-\tanh \beta\left[J^{\lambda} * \underline{M}^{\lambda}+a \lambda\right]\right\} \\
& =\frac{1}{\lambda}\left[\dot{q}\left(\frac{\underline{M}_{0}-\underline{a} t}{\lambda}\right)\left(-\underline{\alpha}+\frac{c^{\lambda}(a)}{\lambda}\right)-\frac{1}{\lambda} A^{\lambda}(x, t) B^{\lambda}(x, t)\right],
\end{aligned}
$$

where

$$
A^{\lambda}(x, t)=\beta \int_{0}^{1} \tanh ^{\prime} \beta\left[\tilde{J} * q+a \lambda+\sigma\left(J^{\lambda} * q-\tilde{J} * q\right)\right] d \sigma
$$

and

$$
B^{\lambda}(x, t)=\int J(|y|)\left\{q\left(\frac{\underline{M}_{0}(x+\lambda y)-\underline{\alpha} t}{\lambda}\right)-q\left(\frac{\underline{M}_{0}(x)-\underline{\alpha} t}{\lambda}+\widehat{D \underline{M}}_{0}(x) \cdot y\right)\right\} d y ;
$$

here $\hat{p}$ denotes the unit vector in the direction of $p, \tilde{J}$ is given by (3.11) and $\dot{q}$ is evaluated at $\lambda^{-1}\left(\underline{M}_{0}(x)-\underline{\alpha} t\right)$.

The properties of $q$ and tanh yield the existence of a constant $b>0$ such that

$$
0 \leqq A^{\lambda}(x, t) \leqq b \text {. }
$$

Rewriting $B^{\lambda}(x, t)$ as

$$
\begin{aligned}
B^{\lambda}(x, t)= & \int J(|y|)\left[q\left(\frac{\underline{M}_{0}(x+\lambda y)-\underline{\alpha} t}{\lambda}\right)-q\left(\frac{\underline{M}_{0}(x)-\underline{\alpha} t}{\lambda}+D \underline{M}_{0}(x) \cdot y\right)\right] d y \\
& +\int J(|y|)\left[q\left(\frac{\underline{M}_{0}(x)-\underline{\alpha} t}{\lambda}+D \underline{M}_{0}(x) \cdot y\right)\right. \\
& \left.-q\left(\frac{\underline{M}_{0}(x)-\underline{\alpha} t}{\lambda}+\widehat{D \underline{M}}_{0}(x) \cdot y\right)\right] d y
\end{aligned}
$$

and using Lemma 5.4 together with (5.9) we get:

$$
\begin{aligned}
B^{\lambda}(x, t) \geqq \lambda & \iint_{0}^{1} J(|y|) \dot{q}\left(\frac{\underline{M}_{0}(x)-\underline{\alpha} t}{\lambda}+D \underline{M}_{0}(x) \cdot y\right. \\
& \left.+\rho \frac{\underline{M}_{0}(x+\lambda y)-\underline{M}_{0}(x)-D \underline{M}_{0}(x) \cdot y}{\lambda}\right) d \rho \\
& \times \int_{0}^{1} \int_{0}^{1}\left(D^{2} \underline{M}_{0}(x+\lambda \sigma \hat{\sigma} y) y, y\right) \sigma d \sigma d \hat{\sigma} d y .
\end{aligned}
$$

Using the above inequality and (5.10) together with the fact that

$$
\dot{q}(\xi+A)=\dot{q}(\xi)(1+O(A)),
$$

which follows immediately from the properties of the traveling wave and, finally, employing (3.12)(iv) and (3.12)(ii), we get

$$
\mathscr{L}\left(\underline{M}^{\lambda}\right) \leqq \frac{1}{\lambda} \dot{q}[-\underline{\alpha}+\bar{b}] \text { for } \lambda \leqq \lambda_{0}(\alpha, \delta),
$$


where the constant $\bar{b}$ depends on the $b$ in (5.10), the constants in (5.11) and (3.12)(iv) and, finally, the second-derivatives of $\underline{M}_{0}$. Choosing $\alpha>\bar{b}$ we conclude

$$
\mathscr{L}\left(\underline{M}^{\lambda}\right) \leqq 0 \text { for } \lambda \leqq \lambda_{0}(a, \delta) .
$$

3. Since, by construction $M_{0} \leqq d_{0}$, we have

$$
\underline{M}^{\lambda} \leqq m^{\lambda} \text { on } \mathbb{R}^{N} \times[0, t] .
$$

The definition of $\underline{M}^{\lambda}$ and (5.2) then yield

$$
\underline{M}_{0}(x)-\underline{\alpha} t \leqq z^{\lambda}(x, t) \text { for }(x, t) \in \mathbb{R}^{N} \times[0, \underline{t}] .
$$

4. A similar argument shows that

$$
z^{\lambda}(x, t) \leqq \bar{M}_{0}(x)-\bar{\alpha} t \text { for }(x, t) \in \mathbb{R}^{N} \times[0, \bar{t}],
$$

where $\bar{M}_{0}$ is an approximation from above of $d_{0}$ and $\bar{\alpha}$ and $\bar{t}$ are chosen appropriately.

5. It is immediate that for all $(x, t) \in \mathbb{R}^{N} \times[0, \underline{t} \wedge \bar{t}]$,

$$
\underline{M}_{0}(x)-\underline{\alpha} t \leqq z_{*}(x, t) \leqq z^{*}(x, t) \leqq \bar{M}_{0}(x)-\bar{\alpha} t,
$$

hence $T>0$.

An immediate consequence of the last inequalities in the proof of Lemma 5.2 is the following lemma.

Lemma 5.5. $z^{*}=z_{*}=d_{0}$ on $\mathbb{R}^{N} \times\{0\}$.

Proof. 1. It is immediate from (4.4), (5.2) and (5.3) that

$$
z^{*} \geqq d_{0} \geqq z_{*} \text { on } \mathbb{R}^{N} \times\{0\} .
$$

2. The last inequalities, in the proof of Lemma 5.2 also yield

$$
\underline{M}_{0} \leqq z_{*} \leqq z^{*} \leqq \bar{M}_{0} \text { on } \mathbb{R}^{N} \times\{0\},
$$

where $\underline{M}_{0}$ and $\bar{M}_{0}$ are approximations of $d_{0}$ from below and above respectively. Letting $\underline{M}_{0} \uparrow d_{0}$ and $\bar{M}_{0} \downarrow d_{0}$, we conclude.

We are finally in a position to proceed with the proof of Lemma 5.3, which again will be split into two parts. Before we go into this, however, we need to introduce some new auxiliary functions.

To this, define

$$
\left\{\begin{array}{l}
\underline{z}^{\lambda}(x, t)=\inf _{y \in \mathbb{R}^{N}}\left\{z^{\lambda}(y, t)+|x-y|\right\} \\
\text { and } \\
\bar{z}^{\lambda}(x, t)=\sup _{y \in \mathbb{R}^{N}}\left\{z^{\lambda}(y, t)-|x-y|\right\} .
\end{array}\right.
$$

The following lemma plays a fundamental role in the analysis below.

Lemma 5.6. Under the assumptions of Theorem 4.1 and for any $T^{\prime} \in(0, T)$, the functions $z^{\lambda}, \underline{z}$ and $z_{*}$ satisfy the following:

(i) $\left|D \underline{z}^{\lambda}\right| \leqq 1$, and $|D \underline{z}| \leqq 1$ in $\mathbb{R}^{N} \times\left[0, T^{\prime}\right]$,

(ii) $\varliminf_{\substack{\lambda \rightarrow 0 \\(y, s) \rightarrow(x, t)}} \underline{z}^{\lambda}(y, s)=\underline{z}(x, t)$ in $\mathbb{R}^{N} \times\left[0, T^{\prime}\right]$, 
(iii) $\operatorname{sgn}\left(z_{*}\right)\left(\left|D z_{*}\right|-1\right) \geqq 0$ in $\mathbb{R}^{N} \times\left[0, T^{\prime}\right]$,

(iv) $|D \underline{z}|=1$ on $\mathbb{R}^{N} \times\left(0, T^{\prime}\right] \cap\{\underline{z}>0\}$,

(v) If $y(x, t) \in \mathbb{R}^{N}$ is such that $\underline{z}(x, t)=z_{*}(y(x, t))+|x-y(x, t)|$ for some $(x, t) \in \mathbb{R}^{N} \times\left[0, T^{\prime}\right] \cap\{\underline{z}>0\}$, then $z_{*}(y(x, t), t)=0$ and

$$
\underline{z}(x, t)=\operatorname{dist}\left(x,\left\{y: z_{*}(y, t)=0\right\}\right) .
$$

The corresponding dual statement holds true for $\bar{z}^{\lambda}, \bar{z}$ and $z^{*}$.

Proof. 1. (i) and (ii) are immediate from the definitions of $\underline{z}^{\lambda}, \underline{z}$ and $z_{*}$.

2. In view of (3.10) and (5.2), we have

$\lambda \dot{q}\left(\frac{z^{\lambda}}{\lambda}\right)\left[z_{t}^{\lambda}-\frac{1}{\lambda} c^{\lambda}(a)\right]+\tanh \beta\left[\tilde{J} * q\left(\frac{z^{\lambda}}{\lambda}\right)+a \lambda\right]-\tanh \beta\left[J * q\left(\frac{z^{\lambda}}{\lambda}\right)+a \lambda\right]=0$,

where to simplify the notation we suppressed the explicit dependence of $q$ on $a \lambda$ and $z^{\lambda}$ is evaluated at $(x, t)$.

After some elementary manipulations, the expression above can be written as:

$$
\begin{aligned}
\lambda \dot{q}\left(z_{t}^{\lambda}-\frac{c^{\lambda}(a)}{\lambda}\right) & -\beta \int_{0}^{1} \tanh ^{\prime}\left[\beta \int J(|y|) q\left(\frac{z^{\lambda}}{\lambda}+e \cdot y\right) d y+a \lambda\right. \\
& +\sigma\left(\beta \int J ( | y | ) \left(q\left(\frac{z^{\lambda}(x+\lambda y)}{\lambda}\right)\right.\right. \\
& \left.\left.-q\left(\frac{z^{\lambda}}{\lambda}+e \cdot y\right) d y\right)\right] d \sigma \\
& \times \int J(|y|) \int_{0}^{1} \dot{q}\left(\frac{z^{\lambda}}{\lambda}+e \cdot y\right. \\
& \left.+\rho\left(\frac{z^{\lambda}(x+\lambda y)}{\lambda}-\frac{z^{\lambda}(x)}{\lambda}-e \cdot y\right)\right) d \rho \\
& \times\left[\frac{z^{\lambda}(x+\lambda y)}{\lambda}-\frac{z^{\lambda}(x)}{\lambda}-e \cdot y\right] d y=0
\end{aligned}
$$

for any unit vector $e$ in $\mathbb{R}^{N}$, where again we suppress the arguments of $z^{\lambda}$ when this does not create any confusion.

3. If $(\underline{x}, \underline{t}) \in \mathbb{R}^{N} \times\left(0, T^{\prime}\right]$ is such that

$$
z_{*}(\underline{x}, \underline{t})>0
$$

then there exists $\left(x_{\hat{\lambda}}, t_{\lambda}\right) \rightarrow(\underline{x}, \underline{t})$ such that $z^{\lambda}\left(x_{\lambda}, t_{\lambda}\right) \rightarrow z_{*}(\underline{x}, \underline{t})$ as $\rightarrow \infty$.

Evaluating the above equality at $\left(x_{\lambda}, t_{\lambda}\right)$, expanding $z^{\lambda}$ around this point up to second order in $x$ and using the asymptotics of $\dot{q}$ and (3.13) as well as the fact that $\int_{0}^{1} \tanh ^{\prime}[\cdot] d \sigma$ is positive and bounded away from zero and sending $\lambda \downarrow 0$, we get

$$
\operatorname{sgn}\left(z_{*}\right) \int J(|y|)\left[e^{-\operatorname{sgn}\left(z_{*}\right) a_{1} D z_{*} \cdot y}-e^{-\operatorname{sgn}\left(z_{*}\right) a_{1} e \cdot y}\right] d y=0
$$


or equivalently

$$
\int J(|y|)\left[e^{-a_{1} D z_{*} \cdot y}-e^{-a_{1} e \cdot y}\right] d y \geqq 0 \text { for all }|e|=1 .
$$

Now pick $e$ such that $D z_{*}=\left|D z_{*}\right| e$ and consider the function

$$
G(\tau)=\int J(|y|)\left[e^{-a_{1} \tau e \cdot y}-e^{-a_{1} e \cdot y}\right] d y .
$$

It is immediate that $G$ is convex, $G(1)=0$ and $G^{\prime}(0)=0$. Hence $G(\tau) \geqq 0$ if and only if $\tau \geqq 1$, thus (5.13) yields

$$
\left|D z_{*}\right| \geqq 1 \text { at }(\underline{x}, \underline{t}) \text {. }
$$

Of course, all the arguments above must be interpreted in the viscosity sensehere to simplify the presentation we chose to argue as if all functions involved were smooth.

An analogous argument in $\left\{z_{*}<0\right\}$ concludes the proof of (iii).

4. If the minimum in (5.4) is achieved at $y(x, t) \neq x$, (iv) is immediate. If $y(x, t)=x$, then (iv) follows from (i) and (iii), since, in this case, $z_{*}(x, t)=$ $\underline{z}(x, t)>0$.

5. We introduce the auxiliary functions $\eta_{n} \in C^{2}(\mathbb{R})$ such that

$$
\eta_{n}(0)=0, \eta_{n}^{\prime}(0)=1, \eta_{n}>-n, 0<\eta^{\prime \prime}<\frac{1}{n} \text { on } \mathbb{R} \text { and } \eta_{n}(\zeta) \downarrow \zeta \text { as } n \rightarrow \infty
$$

and define

$$
\underline{z}_{n}(x, t)=\inf _{y \in \mathbb{R}^{N}}\left\{\eta_{n}\left(z_{*}(y, t)\right)+|x-y|\right\} .
$$

It is immediate that $\underline{z}_{n} \downarrow z$ as $n \rightarrow \infty$ and $\left|D \underline{z}_{n}\right| \leqq 1$ on $\mathbb{R}^{N} \times\left[0, T^{\prime}\right]$. Finally, if $y_{n}(x, t) \in \mathbb{R}^{N}$ is such that

$$
\underline{z}_{n}(x, t)=\eta_{n}\left(z_{*}\left(y_{n}(x, t), t\right)+\left|x-y_{n}(x, t)\right|,\right.
$$

then, passing if necessary to a subsequence,

$$
y_{n}(x, t) \rightarrow y(x, t) \text { and } \underline{z}(x, t)=z_{*}(y(x, t), t)+|x-y(x, t)| .
$$

Observe now that the definitions of $\underline{z}_{n}$ and $y_{n}(x, t)$ yield

$$
\eta_{n}^{\prime}\left(z_{*}\left(y_{n}(x, t), t\right)\right)\left|D z_{*}\left(y_{n}(x, t), t\right)\right| \leqq 1
$$

which in turn, in view of the choice of the $\eta_{n}$ 's, contradicts (iii), unless

$$
z_{*}\left(y_{n}(x, t), t\right) \leqq 0
$$

the case $z_{*}\left(y_{n}(x, t), t\right)<0$ is ruled out similarly using (iv).

All the above need, of course, to be interpreted in the viscosity sense if $t>0$. As a matter of fact (iii) holds in $\left\{z_{*} \neq 0\right\}$; a simple and standard argument in the theory of viscosity solutions then yields that (iii) holds on $\left\{z_{*}(\cdot, t) \neq 0\right\}$ for all $t \in\left(0, T^{\prime}\right]$. Finally, if $t=0$, the conclusion follows from the fact that $z_{*}=d_{0}$ on $\mathbb{R}^{N} \times\{0\}$. 
6. Finally observe that the arguments of steps 4 and 5 above prove the claim for any minimizing $y(x, t)$, which can be obtained as a limit of $y_{n}(x, t)$. On the other hand, a more careful look and routine arguments involving making minima to be strict one's, yields the claim for any minimizing $y(x, t)$.

The last but also very important issue remaining towards completing the proof of Lemma 5.3 is the identification of the transport coefficient $\theta$ in (3.15). This unfortunately cannot be done at the level of the $z^{\lambda}$ s. Instead we need to introduce correctors, as it is done when studying problems in homogenization. This, of course, is not unreasonable, since, as mentioned in Sect. 4, an averaging is indeed taking place at the level of the underlying stochastic process, which corresponds to the linearization of (4.1) around $q$.

Below we introduce the correctors; as usual to simplify the notation we will suppress the explicit dependence of $q$ on $a \lambda$.

For $\mathscr{A}, \mathscr{B} \in \mathbb{R}, \lambda>0$, and for all $e, \hat{e} \in \mathbb{R}^{N}$ such that $|e|=|\hat{e}|=1$ and $e \cdot \hat{e}=0$ a corrector $Q^{\lambda}: \mathbb{R} \rightarrow \mathbb{R}$ is the unique solution of

$$
\begin{aligned}
& \frac{\dot{q}(\xi)}{1-\left(q(\xi)+c^{\lambda}(a) \dot{q}(\xi)\right)^{2}} \mathscr{A}-\frac{\beta}{2}\left[\int J(|y|) \dot{q}(\xi+e \cdot y)(\hat{e} \cdot y)^{2} d y\right] \mathscr{B} \\
& =\beta \int J(|y|) \dot{q}(\xi+e \cdot y)\left[Q^{\lambda}(\xi+e \cdot y)-Q^{\lambda}(\xi)\right] d y
\end{aligned}
$$

with the properties

$$
Q^{\lambda}(0)=0,\left|Q^{\lambda}(\xi)\right| \leqq C|\xi|,\left|\dot{Q}^{\lambda}\right| \leqq C
$$

for some constant $C$. The existence of such a $Q^{\lambda}$ follows - its uniqueness is determined by (5.16) - using Fredholm alternative type of arguments provided

$$
\mathscr{A}=\theta^{\lambda} \mathscr{B},
$$

where

$$
\begin{aligned}
\theta^{\lambda}= & \frac{\beta}{2}\left[\iint J(|y|) \dot{q}(\xi) \dot{q}(\xi+e \cdot y)(\hat{e} \cdot y)^{2} d y d \xi\right] \\
& \times\left[\int \frac{(\dot{q}(\xi))^{2}}{1-\left(q(\xi)+c^{\lambda}(a) \dot{q}(\xi)\right)^{2}} d \xi\right]^{-1} .
\end{aligned}
$$

In view of the properties of the traveling waves $\xi \mapsto q(\xi, a \lambda)$ at the limit $\lambda \rightarrow 0$, it follows that

$$
\theta^{\lambda} \rightarrow \theta \text { as } \lambda \rightarrow 0
$$

with

$$
\theta=\frac{\beta}{2}\left[\iint J(|y|) \dot{q}(\xi) \dot{q}(\xi+e \cdot y)(\hat{e} \cdot y)^{2} d y d \xi\right]\left(\int \frac{(\dot{q}(\xi))^{2}}{1-q^{2}(\xi)} d \xi\right)^{-1}
$$

where now in (5.20),

$$
q(\xi)=q(\xi, 0) .
$$

In preparation for the proof of Lemma 5.3 we state and prove the following lemma; its proof goes along the lines of analogous results in [BSS], which, however, need to be modified to account for the correctors. 
Lemma 5.7. Under the assumptions of Lemma 5.3, $\underline{z}$ (resp. $\bar{z}$ ) is a super- (resp. sub-) solution of (3.17) with $A=c(a)$ in $\{\underline{z}>0\} \cap \mathbb{R}^{N} \times\left(0, T^{\prime}\right)($ resp. $\{\bar{z}<0\} \cap$ $\left.\mathbb{R}^{N} \times\left(0, T^{\prime}\right)\right)$.

Proof. 1. Let $\left(x_{0}, t_{0}\right) \in\{\underline{z}>0\} \cap \mathbb{R}^{N} \times\left(0, T^{\prime}\right)$ be a strict minimum of $\underline{z}-\phi$, for some smooth test function $\phi$. Without any loss of generality, we may assume

$$
\underline{z} \geqq \phi \text { on } \mathbb{R}^{N} \times\left[0, T^{\prime}\right] \text { with equality only at }\left(x_{0}, t_{0}\right) \text {. }
$$

We want to show

$$
\phi_{t}-\theta \operatorname{tr}\left[I-\frac{D \phi \otimes D \phi}{|D \phi|^{2}}\right] D^{2} \phi-c(a)|D \phi| \geqq 0 \text { at }\left(x_{0}, t_{0}\right) .
$$

2. In view of Lemma 5.6 (iv), we have

$$
\left|D \phi\left(x_{0}, t_{0}\right)\right|=1 \text {. }
$$

3. Consider the corrector $Q^{\lambda}$ defined by (5.15) and (5.17) for

$$
\mathscr{A}=\phi_{t}\left(x_{0}, t_{0}\right)-c(a) \text { and } \mathscr{B}=\frac{1}{\theta^{\lambda}} \mathscr{A},
$$

where $\theta^{\lambda}$ is given by (5.18). Again recall that we will be writing $q$ for $q(\cdot, a \lambda)$ throughout this proof.

4. In view of the properties of $Q^{\lambda}$, the map $\xi \rightarrow \xi+\lambda^{2} Q^{\lambda}(\xi / \lambda)$ is $1-1$ and onto, hence we can write

$$
z^{\lambda}=w^{\lambda}+\lambda^{2} Q^{\lambda}\left(\frac{w^{\lambda}}{\lambda}\right) \text { on } \mathbb{R}^{N} \times\left[0, T^{\prime}\right]
$$

for some functions $w^{\lambda}: \mathbb{R}^{N} \times\left[0, T^{\prime}\right] \rightarrow \mathbb{R}$ and

$$
m^{\lambda}=q\left(\frac{w^{\lambda}}{\lambda}+\lambda Q^{\hat{\lambda}}\left(\frac{w^{\lambda}}{\lambda}\right)\right) \text { on } \mathbb{R}^{N} \times\left[0, T^{\prime}\right] .
$$

It is immediate that

$$
w_{*}=z_{*} \text { and } w^{*}=z^{*} \text {. }
$$

Define

$$
\underline{w}^{\lambda}(x, t)=\inf _{y \in \mathbb{R}^{N}}\left\{w^{\lambda}(y, t)+|x-y|\right\} .
$$

Our assumptions on $T^{\prime}$ immediately deliver, for all $(x, t) \in \mathbb{R}^{N} \times\left[0, T^{\prime}\right]$,

$$
\varliminf_{\substack{\lambda \rightarrow 0 \\(y, s) \rightarrow(x, t)}} \underline{w}^{\lambda}(y, s)=\underline{z}(x, t) .
$$

5. Lemma 5.6, (5.22) and (5.30) yield the existence of and $\left(x^{\lambda}, t^{\lambda}\right) \rightarrow\left(x_{0}, t_{0}\right)$ as $\lambda \rightarrow 0$, such that

$$
\left(x^{\lambda}, t^{\lambda}\right) \text { is a minimum of } \underline{w}^{\lambda}-\phi \text { and } \underline{w}^{\lambda}\left(x^{\lambda}, t^{\lambda}\right) \rightarrow \underline{z}\left(x_{0}, t_{0}\right) ;
$$

actually (5.30) yields the existence of $\left(x^{\lambda_{m}}, t^{\lambda_{m}}\right) \rightarrow\left(x_{0}, t_{0}\right)$ along a sequence $\lambda_{m} \rightarrow 0$, but for notational simplicity we only write $\lambda \rightarrow 0$ below. 
Let $\left(y^{\lambda}, t^{\lambda}\right)$ and $\left(y_{0}, t_{0}\right)$ be points at which the inf is attained in (5.29) and (5.12) respectively. Without any loss of generality we may assume that

$$
\left\{\begin{array}{l}
\left(y^{\lambda}, t^{\lambda}\right) \rightarrow\left(y_{0}, t_{0}\right) \text { as } \lambda \rightarrow 0 \\
\text { and } \\
w^{\lambda} \geqq \Psi^{\lambda} \text { with equality at }\left(y^{\lambda}, t^{\lambda}\right),
\end{array}\right.
$$

where

$$
\Psi^{\lambda}(x, s)=\phi\left(x+x^{\lambda}-y^{\lambda}, s\right) .
$$

Using now Lemma 5.6(v) and (5.28), it is easy to see that

$$
\lim _{\lambda \rightarrow 0} w^{\lambda}\left(y^{\lambda}, t^{\lambda}\right)=0 \text {. }
$$

Arguing as in Step 4 of the proof of Lemma 5.6 we also get

$$
\operatorname{sgn}\left(w^{\lambda}\left(y^{\lambda}, t^{\lambda}\right)\right)\left(\left|D w^{\lambda}\left(y^{\lambda}, t^{\lambda}\right)\right|-1\right) \leqq 0,
$$

and using (5.24) and (5.34),

$$
\left|D \Psi^{\lambda}\left(y^{\lambda}, t^{\lambda}\right)\right|=\left|D \phi\left(x^{\lambda}, t^{\lambda}\right)\right| \rightarrow 1 \text { as } \lambda \rightarrow 0,
$$

the latter following from (5.31) and (5.24).

6. We now turn to the equation satisfied by the $w^{\lambda}$ 's, which we evaluate at $\left(y^{\lambda}, t^{\lambda}\right)$. In everything that follows below to simplify the notation we will not be writing the arguments of $z^{\lambda}$ and $w^{\lambda}$ and almost never write the $t$-argument, unless it is necessary. We will also write $q, \dot{q}, \ddot{q}$ and $Q, \dot{Q}$ without their arguments, which are $z^{\lambda} / \lambda$ and $w^{\lambda} / \lambda$ respectively - recall $z^{\lambda}=w^{\lambda}+\lambda^{2} Q\left(w^{\lambda} / \lambda\right)$ - and as before we will not exhibit the dependence of $q$ and $Q$ on $\lambda$. Finally, all the calculations below must be interpreted in the viscosity sense. On the other hand, keeping in mind that eventually the paper must end at some point, we present them as if everything were smooth.

A simple computation and Eq. (3.10) yields, at $\left(y^{\lambda}, t^{\lambda}\right)$ :

$$
\begin{gathered}
\dot{q}\left[\left(\frac{1}{\lambda}+\dot{Q}\right) w_{t}^{\lambda}-\frac{1}{\lambda^{2}} c^{\lambda}(a)\right]-\frac{1}{\lambda^{2}}\left\{\operatorname { t a n h } \left[\beta\left[J^{\lambda} * q+a \lambda\right]\right.\right. \\
-\tanh [\beta[(\tilde{J} * q)+a \lambda]\}=0 .
\end{gathered}
$$

Rewrite the above equality as

$$
\begin{aligned}
\dot{q} & {\left[\left(\frac{1}{\lambda}+\dot{Q}\right) w_{t}^{\lambda}-\frac{1}{\lambda^{2}} c^{\lambda}(a)\right]-\frac{1}{\lambda^{2}}\left\{\tanh \left[\beta\left(J^{\lambda} * q+a \lambda\right)\right]\right.} \\
& \left.-\tanh \left[\beta\left(\int J(|y|) q\left(\frac{w^{\lambda}}{\lambda}+\lambda Q\left(\frac{w^{\lambda}}{\lambda}\right)+D w^{\lambda} \cdot y\right) d y+a \lambda\right)\right]\right\} \\
\geqq & \frac{1}{\lambda^{2}}\left\{\tanh \left[\beta\left(\int J(|y|) q\left(\frac{z^{\lambda}}{\lambda}+D w^{\lambda} \cdot y\right)+a \lambda\right) d y\right]\right. \\
& \left.-\tanh \left[\beta\left(\int J(|y|) q\left(\frac{z^{\lambda}}{\lambda}+\widehat{D w^{\lambda}} \cdot y\right) d y+a \lambda\right)\right]\right\},
\end{aligned}
$$

where $\widehat{D w^{\lambda}}$ is the unit vector in the direction of $D w^{\lambda}$. 
The inequality in (5.37) is not a misprint. It is there, due to the ellipticity of the nonlocal equation, when we use the test functions $\Psi$ instead of the $w^{\lambda}$ 's.

7. Set $\widehat{D w^{\lambda}}=e$ and write $D w^{\lambda}=\tau e$. In view of (5.35) we have

$$
\operatorname{sgn}\left(w^{\lambda}\right)(\tau-1) \leqq 0 .
$$

On the other hand, since $\xi \mapsto \xi+\lambda^{2} Q(\xi / \lambda)$ is $1-1$ and $Q(0)=0$,

$$
\operatorname{sgn}\left(w^{\lambda}\right)=\operatorname{sgn}\left(z^{\lambda}\right) .
$$

Applying Lemma 5.4 with $\xi=z^{\lambda} / \lambda$ and using the above, we see that the expression in the right-hand side of (5.37) is nonnegative.

Hence

$$
\begin{aligned}
& \dot{q}\left[\left(\frac{1}{\lambda}+\dot{Q}\right) w_{t}^{\lambda}-\frac{1}{\lambda^{2}} c^{\lambda}(a)\right]-\frac{1}{\lambda^{2}}\left\{\tanh \left[\beta\left(J^{\lambda} * q+a \lambda\right)\right]\right. \\
& \left.\quad-\tanh \left[\beta\left(\int J(|y|) q\left(\frac{w^{\lambda}}{\lambda}+\lambda Q+D w^{\lambda} \cdot y\right) d y+a \lambda\right)\right]\right\} \geqq 0,
\end{aligned}
$$

and, after some elementary calculus,

$$
\begin{aligned}
\dot{q} & {\left[\left(\frac{1}{\lambda}+\dot{Q}\right) w_{t}^{\lambda}-\frac{c^{\lambda}(a)}{\lambda^{2}}\right] } \\
& -\frac{1}{\lambda^{2}} A^{\lambda}\left\{J^{\lambda} * q-\int J(|y|) q\left(\frac{w^{\lambda}}{\lambda}+\lambda Q+D w^{\lambda} \cdot y\right) d y\right\} \geqq 0,
\end{aligned}
$$

where

$$
\begin{aligned}
A^{\lambda}= & \int_{0}^{1} \beta \tanh ^{\prime}\left\{\beta \left[\int J(|y|) q\left(\frac{w^{\lambda}}{\lambda}+\lambda Q+D w^{\lambda} \cdot y\right) d y+a \lambda\right.\right. \\
& \left.\left.+\sigma\left[J^{\lambda} * q-\int J(|y|) q\left(\frac{w^{\lambda}}{\lambda}+\lambda Q+D w^{\lambda} \cdot y\right) d y\right]\right]\right\} d \sigma .
\end{aligned}
$$

8. We concentrate for the moment on the term multiplying $A^{\lambda}$ in (5.38), which for definiteness we call $B^{\lambda}$ :

$$
\begin{aligned}
B^{\lambda}= & J^{\lambda} * q-\int J(|y|) q\left(\frac{w^{\lambda}}{\lambda}+\lambda Q+D w^{\lambda} \cdot y\right) d y \\
= & \int J(|y|)\left\{q\left(\frac{w^{\lambda}\left(y^{\lambda}+\lambda y\right)}{\lambda}+\lambda Q\left(\frac{w^{\lambda}\left(y^{\lambda}+\lambda y\right)}{\lambda}\right)\right)\right. \\
& \left.-q\left(\frac{w^{\lambda}\left(y^{\lambda}\right)}{\lambda}+\lambda Q\left(\frac{w^{\lambda}\left(y^{\lambda}\right)}{\lambda}\right)+D w^{\lambda} \cdot y\right)\right\} d y \\
= & \int J(|y|) \int_{0}^{1} \dot{q}\left(\frac{w^{\lambda}\left(y^{\lambda}\right)}{\lambda}+\lambda Q\left(\frac{w^{\lambda}\left(y^{\lambda}\right)}{\lambda}\right)+D w^{\lambda} \cdot y+\rho C^{\lambda}\right) d \rho C^{\lambda} d y,
\end{aligned}
$$


with

$$
\begin{aligned}
C^{\lambda}= & \frac{w^{\lambda}\left(y^{\lambda}+\lambda y\right)}{\lambda}+\lambda Q\left(\frac{w^{\lambda}\left(y^{\lambda}+\lambda y\right)}{\lambda}\right) \\
& -\frac{w^{\lambda}\left(y^{\lambda}\right)}{\lambda}-\lambda Q\left(\frac{w^{\lambda}\left(y^{\lambda}\right)}{\lambda}\right)-D w^{\lambda} \cdot y \\
= & \left.\lambda\left\{\frac{1}{2}\left(D^{2} w^{\lambda}\left(y^{\lambda}\right) y, y\right)+Q\left(\frac{w^{\lambda}\left(y^{\lambda}\right)}{\lambda}+D w^{\lambda} \cdot y\right)-Q\left(\frac{w^{\lambda}\left(y^{\lambda}\right)}{\lambda}\right)\right)\right\} \\
& +O\left(\lambda^{2}\right)+\lambda\left\{Q\left(\frac{w^{\lambda}\left(y^{\lambda}\right)}{\lambda}+D w^{\lambda} \cdot y+O(\lambda)\right)\right. \\
& \left.-Q\left(\frac{w^{\lambda}\left(y^{\lambda}\right)}{\lambda}+D w^{\lambda} \cdot y\right)\right\} .
\end{aligned}
$$

Notice that the last curly bracket in $C^{\lambda}$ is of order $O(\lambda)$, since $|\dot{Q}| \leqq C$. Finally, we remind the reader that all the above calculations are done for the smooth test function $\Psi^{\lambda}$ and not for $w^{\lambda}$, hence we obtain the $O(\lambda)$ in $C^{\lambda}$ by expanding the smooth test function.

It is also clear that

$$
C^{\lambda}=O(\lambda)
$$

The properties of $Q$ and (5.34) also yield

$$
\lambda Q\left(\frac{w^{\lambda}\left(y^{\lambda}, t^{\lambda}\right)}{\lambda}\right)=o(1) .
$$

In addition, (3.12) implies, for some constant $C$ and all $\xi \in \mathbb{R}$,

$$
\left|\frac{\ddot{q}(\xi)}{\dot{q}(\xi)}\right| \leqq C \text { and } \frac{\dot{q}(\xi+o(1))}{\dot{q}(\xi)}=1+o(1) .
$$

Combining all the above and once more (3.12) we obtain

$$
B^{\lambda}=\dot{q}\left(\frac{w^{\lambda}}{\lambda}\right) o(1)
$$

Finally, using (5.36), (5.42), (5.43) and (5.44) we conclude

$$
\begin{gathered}
\dot{q}\left(\frac{w^{\lambda}\left(y^{\lambda}\right)}{\lambda}+\lambda Q\left(\frac{w^{\lambda}\left(y^{\lambda}\right)}{\lambda}\right)+D w^{\lambda} \cdot y+\rho C^{\lambda}\right) \\
=\dot{q}\left(\frac{w^{\lambda}}{\lambda}+\widehat{D w^{\lambda}} \cdot y\right)(1+o(1)) .
\end{gathered}
$$


8. We now return to (5.39). A combination of (5.36), (5.40), (5.42) and (5.45) delivers

$$
\begin{aligned}
A^{\lambda} & =\int_{0}^{1} \beta \tanh ^{\prime}\left\{\beta\left[\int J(|y|) q\left(\frac{w^{\lambda}}{\lambda}+\lambda Q+D w^{\lambda} \cdot y\right) d y+a \lambda+\sigma B^{\lambda}\right]\right\} d \sigma \\
& =\beta \tanh ^{\prime}\left\{\beta\left[\int J(|y|) q\left(\frac{w^{\lambda}}{\lambda}+\widehat{D w^{\lambda}} \cdot y+o(1)\right) d y+a \lambda+\dot{q}\left(\frac{w^{\lambda}}{\lambda}\right) o(1)\right]\right\} \\
& =\beta \tanh ^{\prime}\left\{\beta\left[\tilde{J} * q\left(\frac{w^{\lambda}}{\lambda}\right)+a \lambda\right]\right\}+\dot{q}\left(\frac{w^{\lambda}}{\lambda}\right) o(1) \\
& =\beta\left\{1-\left[q\left(\frac{w^{\lambda}}{\lambda}\right)+c^{\lambda}(a) \dot{q}\left(\frac{w^{\lambda}}{\lambda}\right)\right]^{2}\right\}+\dot{q}\left(\frac{w^{\lambda}\left(y^{\lambda}, t^{\lambda}\right)}{\lambda}\right) o(1) .
\end{aligned}
$$

Notice that in the above expression $q$ and $\dot{q}$ are now evaluated at $w^{\lambda} / \lambda$ instead of $z^{\lambda} / \lambda$.

Now using (5.41) and (5.46), $B^{\hat{\lambda}}$ becomes

$$
\begin{aligned}
B^{\lambda}= & \int J(|y|) \dot{q}\left(\frac{w^{\lambda}}{\lambda}+\widehat{D w^{\lambda}} \cdot y\right)(1+o(1))\left\{\lambda \left[\frac{1}{2}\left(D^{2} w^{\lambda} y, y\right)\right.\right. \\
& \left.\left.+Q\left(\frac{w^{\lambda}}{\lambda}+\widehat{D w^{\lambda}} \cdot y\right)-Q\left(\frac{w^{\lambda}}{\lambda}\right)\right] d y+o(\lambda)\right\} d y .
\end{aligned}
$$

Substituting (5.47) and (5.48) in (5.38) and using (3.12)(ii) we get

$$
\begin{aligned}
& \dot{q}\left(\frac{w^{\lambda}}{\lambda}\right)(1+o(1))\left[[1+\lambda \dot{Q}]\left(w_{t}^{\lambda}-\frac{1}{\lambda} c^{\lambda}(a)\right)\right. \\
& \quad-\frac{1}{\lambda}\left[\beta\left\{1-\left[q\left(\frac{w^{\lambda}}{\lambda}\right)+c^{\lambda}(a) \dot{q}\left(\frac{w^{\lambda}}{\lambda}\right)\right]^{2}\right\}+\dot{q}\left(\frac{w^{\lambda}}{\lambda}\right) o(1)\right] \\
& \quad \times \int J(|y|) \dot{q}\left(\frac{w^{\lambda}}{\lambda}+\widehat{D w^{\lambda}} \cdot y\right)(1+o(1))\left\{\lambda \left[\frac{1}{2}\left(D^{2} w^{\lambda} y, y\right)\right.\right. \\
& \left.\left.\quad+Q\left(\frac{w^{\lambda}}{\lambda}+\widehat{D w^{\lambda}} \cdot y\right)-Q\left(\frac{w^{\lambda}}{\lambda}\right)\right]+o(\lambda)\right\} d y \geqq 0,
\end{aligned}
$$

where the last inequality is of course interpreted in the viscosity sense.

9. Replacing $w^{\lambda}$ by $\Psi^{\lambda}$ (see (5.33)) and making the obvious simplifications we obtain:

$$
\begin{aligned}
& \frac{\dot{q}\left(\frac{w^{i}\left(y^{i}, t^{i}\right)}{\lambda}\right)}{1-\left[q\left(\frac{w^{j}}{\lambda}\right)+c^{\lambda}(a) \dot{q}\left(\frac{w^{j}}{\lambda}\right)\right]^{2}}\left[\phi_{t}\left(x^{\lambda}, t^{\lambda}\right)-c(a)\right] \\
& -\beta \int J(|y|) \dot{q}\left(\frac{w^{\lambda}\left(y^{\lambda}, t^{\lambda}\right)}{\lambda}+\widehat{D \Psi}\left(x^{\lambda}, t^{\lambda}\right) \cdot y\right) \times\left[\frac{1}{2}\left(D^{2} \Psi\left(x^{\lambda}, t^{\lambda}\right) y, y\right)\right] \\
& +\left[Q\left(\frac{w^{\lambda}\left(y^{\lambda}, t^{\lambda}\right)}{\lambda}+\widehat{D \Psi}\left(x^{\lambda}, t^{\lambda}\right) \cdot y\right)-Q\left(\frac{w^{\lambda}\left(y^{\lambda}, t^{\lambda}\right)}{\lambda}\right)+o(1)\right] d y \\
& +\dot{q}\left(\frac{w^{\lambda}}{\lambda}\right) o(1) \geqq 0 .
\end{aligned}
$$


We may now replace $\Psi_{t}\left(x^{\hat{\lambda}}, t^{\lambda}\right), D \Psi\left(x^{\hat{\lambda}}, t^{\lambda}\right)$ and $D^{2} \Psi\left(x^{\lambda}, t^{\lambda}\right)$ by $\Psi_{t}\left(x_{0}, t_{0}\right)$, $D \Psi\left(x_{0}, t_{0}\right)$ and $D^{2} \Psi\left(x_{0}, t_{0}\right)$ respectively by making an error, (see (5.44)), of the order $\dot{q}\left(\frac{w^{\prime}\left(y^{\prime}, t^{\prime}\right)}{i}\right) o(1)$.

Recall now that $Q$ solves

$$
\begin{aligned}
& \frac{\dot{q}(\xi)}{1-\left(q(\xi)+c^{\lambda}(a) \dot{q}(\xi)\right)^{2}}\left[\phi_{t}\left(x_{0}, t_{0}\right)-c(a)\right] \\
& -\frac{\beta}{2}\left[\int J(|y|) \dot{q}(\xi+e \cdot y)(\hat{e} \cdot y)^{2} d y\right] \frac{1}{\theta^{\lambda}}\left(\phi_{t}\left(x_{0}, t_{0}\right)-c(a)\right) \\
& \quad=\beta \int J(|y|) \dot{q}(\xi+e \cdot y)[Q(\xi+e \cdot y)-Q(\xi)] d y,
\end{aligned}
$$

for

$$
e=D \phi\left(x_{0}, t_{0}\right) \text { and some } \hat{e} \in \mathbb{R}^{N} \text { such that }|\hat{e}|=1 \text { and } e \cdot \hat{e}=0 .
$$

Substituting in the previous inequality we get

$$
\begin{aligned}
& \frac{\beta}{2} \int J(|y|) \dot{q}\left(\frac{w^{\lambda}}{\lambda}+D \phi\left(x_{0}, t_{0}\right) \cdot y\right)\left[(\hat{e} \cdot y)^{2} \frac{1}{\theta}\left[\phi_{t}\left(x_{0}, t_{0}\right)-c(a)\right]\right. \\
& \left.-\left(D^{2} \phi\left(x_{0}, t_{0}\right) y, y\right)\right] d y \geqq o(1) \dot{q}\left(\frac{w^{\lambda}}{\lambda}\right),
\end{aligned}
$$

where $w^{\lambda}$ is evaluated at $\left(y^{\lambda}, t^{\lambda}\right)$.

10 . We now turn to the term

$$
\int J(|y|) \dot{q}\left(\xi+D \phi\left(x_{0}, t_{0}\right) \cdot y\right)\left(D^{2} \phi\left(x_{0}, t_{0}\right) y, y\right) d y .
$$

Assuming for simplicity, in (5.50) $D \phi\left(x_{0}, t_{0}\right)=e_{n}$, writing $y=\sum_{i=1}^{n} y_{i} e_{i}$ and using the symmetry of $J$, for any $\xi \in \mathbb{R}$, we obtain

$$
\begin{aligned}
\int J(|y|) \dot{q}\left(\xi+e_{n} \cdot y\right)\left(D^{2} \phi\left(x_{0}, t_{0}\right) y, y\right) d y= & \sum_{i=1}^{n-1} \int J(|y|) \dot{q}\left(\xi+e_{n} \cdot y\right) y_{1}^{2} \phi_{x_{1} x_{l}}\left(x_{0}, t_{0}\right) d y \\
& \left.+\int J(|y|) \dot{q}\left(\xi+e_{n} \cdot y\right)\right) y_{n}^{2} \phi_{x_{n} x_{n}}\left(x_{0}, t_{0}\right) d y .
\end{aligned}
$$

Inserting the above identity with $\xi=\lambda^{-1} w^{\lambda}\left(y^{\lambda}, t^{\lambda}\right)$ in (5.50), yields

$$
\begin{aligned}
& \frac{\beta}{2} \int J(|y|) \dot{q}\left(\frac{w^{\lambda}}{\lambda}+D \phi\left(x_{0}, t_{0}\right) \cdot y\right)(\hat{e} \cdot y)^{2} d y\left\{\frac{1}{\theta} \phi_{t}\left(x_{0}, t_{0}\right)\right. \\
& \left.-\operatorname{tr}\left(I-\frac{D \phi \otimes D \phi}{|D \phi|^{2}}\right) D^{2} \phi\left(x_{0}, t_{0}\right)\right\}-\frac{\beta}{2} \int J(|y|) \dot{q}\left(\frac{w^{\lambda}}{\lambda}+D \phi\left(x_{0}, t_{0}\right) \cdot y\right) \\
& \quad\left(e_{n} \cdot y\right)^{2} d y\left\{\left(D^{2} \phi D \phi\left(x_{0}, y_{0}\right), D \phi\left(x_{0}, t_{0}\right)\right\} \geqq o(1) \dot{q}\left(\frac{w^{\lambda}}{\lambda}\right),\right.
\end{aligned}
$$

where again $w^{\lambda}$ is evaluated at $\left(y^{\lambda}, t^{\lambda}\right)$.

Recalling (see $3.10(\mathrm{v})$ ) that for all $\xi \in \mathbb{R}$,

$$
0<a_{3}^{-1} \leqq \frac{\int J(|y|) \dot{q}(\xi+e \cdot y)(e \cdot y)^{2} d y}{\dot{q}(\xi)} \leqq a_{3}
$$


and

$$
0<a_{3}^{-1} \leqq \frac{\int J(|y|) \dot{q}(\xi+e \cdot y)(\hat{e} \cdot y)^{2} d y}{\dot{q}(\xi)} \leqq a_{3},
$$

as well as the Lipschitz continuity of $Q$, we let $\lambda \downarrow 0$ and obtain, for some $\mu>0$,

$$
\underline{z}_{t}-\theta \operatorname{tr}\left(1-\frac{D \underline{z} \otimes D \underline{z}}{|D \underline{z}|^{2}}\right) D^{2} \underline{z}-c(a)|D \underline{z}| \geqq \mu\left(D^{2} \underline{z} D \underline{z}, D \underline{z}\right) \text { in }\{\underline{z}>0\} \text {. }
$$

To conclude we argue exactly as in the proof of Lemma 5.2 of [BSS] in order to drop the right-hand side of (5.52). This is based on the observation that since $|D \underline{z}|=1$ in $\{\underline{z}>0\}$, then roughly speaking

$$
D^{2} \underline{z} D \underline{z}=0 \quad \text { a.e. }
$$

For all the details we refer the reader to the proof of Lemma 5.2 of [BSS].

11. The arguments for $\bar{z}$ are similar.

We are finally ready for the proof of Lemma 5.3.

Proof of Lemma 5.3. 1. For $\delta>0$ consider the smooth functions $\psi_{\delta}: \mathbb{R} \rightarrow \mathbb{R}$ such that

$$
\psi_{\delta}=0 \text { on }(-\infty,-\delta], \psi_{\delta}^{\prime} \geqq 0 \text { in } \mathbb{R} \text { and } \lim _{\delta \rightarrow 0} \psi_{\delta}(s)=s^{+} \text {, }
$$

and define

$$
v_{\delta}=\psi_{\delta}(\underline{z}) .
$$

2. Let $\left(x_{0}, t_{0}\right) \in \mathbb{R}^{N} \times\left(0, T^{\prime}\right)$ be a strict minimum of $v_{\delta}-\phi$ for some smooth function $\phi$. If $\underline{z}\left(x_{0}, t_{0}\right)>0$, we conclude easily using the fact that (3.15) is geometric, i.e. invariant under increasing changes.

Let now $\left(x_{0}, t_{0}\right) \in\{\underline{z} \leqq 0\} \cap \mathbb{R}^{N} \times\left(0, T^{\prime}\right)$. The Lipschitz continuity of $v_{\delta}$ in $x$ implies that $v_{\delta}=0$ in a ball around $x_{0}$, hence

$$
D \phi\left(x_{0}, t_{0}\right)=0 \text {. }
$$

In this case we may assume

$$
D^{2} \phi\left(x_{0}, t_{0}\right)=0,
$$

hence we only need to show that

$$
\phi_{t}\left(x_{0}, t_{0}\right) \geqq 0
$$

Set

$$
v_{\delta}^{\lambda}(y, s)=\psi_{\gamma}\left(\underline{z}^{\lambda}\right)
$$

and notice that

$$
\varliminf_{\substack{(y, s) \rightarrow(x, t) \\ \lambda \rightarrow 0}} v_{\delta}^{\lambda}(y, s)=v_{\delta}(x, t) .
$$


Thus $v_{\delta}^{\lambda}-\phi$ has a local minimum at $\left(x^{\lambda}, t^{\lambda}\right)$ such that $\left(x^{\lambda}, t^{\lambda}\right) \rightarrow\left(x_{0}, t_{0}\right)$ as $\lambda \rightarrow 0$. Furthermore, using (5.49) with $Q \equiv 0$ we get

$$
\begin{aligned}
& \dot{q}\left(\frac{z^{\lambda}}{\lambda}\right)\left[\phi_{t}\left(x^{\lambda}, t^{\lambda}\right)-c(a)+o(1)\right] \\
& \quad \geqq a^{\lambda}\left(x^{\lambda}, y^{\lambda}, t^{\lambda}\right) \int J(|y|) \dot{q}\left(\frac{z^{\lambda}}{\lambda}+e \cdot y\right)\left\{\frac{1}{2}\left(D^{2} \phi\left(x^{\lambda}, t^{\lambda}\right) y, y\right)+o(1)\right\} d y,
\end{aligned}
$$

where $a^{\lambda}\left(x^{\lambda}, y^{\lambda}, t^{\lambda}\right)>0$ and $z^{\lambda}$ is evaluated at $\left(y^{\lambda}, t^{\lambda}\right)$.

Sending $\lambda \downarrow 0$ we obtain

$$
\phi_{t}\left(x_{0}, t_{0}\right) \geqq 0
$$

The above yield

$$
\phi_{t}-\theta \operatorname{tr}\left(I-\frac{D \phi \otimes D \phi}{|D \phi|^{2}}\right) D^{2} \phi-c(a)|D \phi| \geqq 0 \text { at }\left(x_{0}, t_{0}\right),
$$

hence $v_{\delta}$ is a supersolution in $\{\underline{z} \geqq 0\}$. We conclude letting $\delta \downarrow 0$.

\section{Proof and Corollaries of Theorem 4.2}

(i) Proof of Theorem 4.2. As before we organize the proof of Theorem 4.2 in a series of lemmas, which we give and prove below. At the end of the section we also state some immediate corollaries.

At this point we want to remind the reader that we return to the convention of Sects. 2-4 denoting points in $\mathbb{Z}^{N}$ by $x, y$, etc. and points in $\mathbb{R}^{N}$ by $r, r^{\prime}$, etc. Finally throughout this section we will denote all uniform constants by $C$, keeping of course in mind that they may change from place to place.

An important ingredient in the proof will be the mean field equation

$$
m_{t}+m-\tanh \beta(J * m)=0 \quad \text { in } \quad \mathbb{R}^{N} \times(0, \infty),
$$

and its discretized version

$$
m_{t}^{\gamma}+m^{\gamma}-\tanh \beta\left(J_{\gamma} \circ m^{\gamma}\right)=0 \quad \text { on } \quad \mathbb{Z}^{N} \times(0, \infty)
$$

where $J_{\gamma} \circ f$ is the discrete convolution on $\mathbb{Z}^{N}$, i.e.

$$
J_{\gamma} \circ f(x)=\sum_{\substack{y \in \mathbb{Z}^{N} \\ y \neq 1}} J_{\gamma}(x, y) f(y) .
$$

We write $m(\cdot, \cdot \mid \sigma)$ and $m^{\gamma}(\cdot, \cdot \mid \sigma)$ to denote the solutions of (6.1) and (6.2) respectively with initial datum

$$
m(r, 0 \mid \sigma)=g(r) \quad\left(r \in \mathbb{R}^{N}\right)
$$

and

where, for $x \in \mathbb{Z}^{N}$,

$$
m^{\gamma}(x, 0 \mid \sigma)=\sigma(x) \quad\left(x \in \mathbb{Z}^{N}\right)
$$

$$
g(\gamma x)=\sigma(x)
$$

respectively. 
The first lemma estimates the difference of the solutions of the discrete and continuous mean field equations. Since its proof is a straightforward application of Gronwall's inequality, we omit it.

Lemma 6.1. There exists a constant $C>0$ such that for all $t>0$,

$$
\sup _{x \in \mathbb{Z}^{N}}\left|m^{\gamma}(x, t \mid \sigma)-m(\gamma x, t \mid \sigma)\right| \leqq C e^{c t} \gamma
$$

Next we state an estimate on the fluctuations of the stochastic process $\left\{\sigma_{t}\right\}_{t \geqq 0}$ around the solution $m^{\gamma}(\cdot, \cdot \mid \sigma)$ of $(6.2)$ in terms of the correlation functions, also known as the $v$-functions,

$$
v_{n}^{\gamma}(\underline{x}, t \mid \sigma)=\mathbb{E}_{\sigma}\left\{\prod_{x \in \underline{x}}\left[\sigma_{t}(x)-m^{\gamma}(x, t \mid \sigma)\right]\right\},
$$

where, for each $n \in \mathbb{N}, \underline{x} \in \mathbb{Z}_{n}^{N}$ - see (2.4) for the definition of $\mathbb{Z}_{n}^{N}$. The following estimate on the $v$-functions is proved in [DOPT1] under the condition that the potential $J$ is $C^{3}$. A careful examination of its proof, however, reveals that (2.1) is enough.

Lemma 6.2. Assume (2.1). Then for any $\delta>0$ and $n \geqq 1$ there is a constant $c_{n}>0$ such that

$$
\sup _{x \in \mathbb{Z}_{n}^{N}} \sup _{t \in\left[0, \gamma^{\delta}\right]} \sup _{\sigma \in \Sigma}\left|v_{n}^{\gamma}(\underline{x}, t \mid \sigma)\right| \leqq c_{n} \gamma^{\frac{N n}{2}}
$$

It is important to have an $L^{\infty}$-norm type estimate on the difference $\sigma_{t}$ $m^{\gamma}(\cdot, t \mid \sigma)$. Lemma 6.2, however, does not yield such an estimate, since it does not allow a straightforward application of Chebyshev's inequality. To overcome this difficulty, following [DP, B, KS], etc., we introduce the following $\gamma$-seminorm on $\mathbb{Z}^{N}$ :

$$
\|f\|_{\gamma, S}=\sup _{x \in S}\left|T^{\gamma}\left(\gamma^{\xi}\right) f(x)\right|
$$

where

$$
\xi>\delta
$$

with $\delta$ as in Lemma 6.2. Here, $S$ is a subset of $\mathbb{Z}^{N}$ and $t \mapsto T^{\gamma}(t)$ is the semigroup associated with the equation

$$
u_{t}^{\gamma}=\alpha_{\gamma} u^{\gamma}+\beta\left(J_{\gamma} \circ u^{\gamma}-\hat{J}_{\gamma, 0} u^{\gamma}\right) \text { in } \mathbb{Z}^{N} \times(0, \infty),
$$

where

$$
\alpha_{\gamma}=\beta \hat{J}_{\gamma, 0}-1 \text { and } \hat{J}_{\gamma, 0}=\sum_{y} J_{\gamma}(0, y)
$$

which is the linearization of (6.2) around $m^{\gamma} \equiv 0$. For a given $f \in L^{\infty}\left(\mathbb{Z}^{N}\right)$,

$$
T^{\gamma}(t) f(x)=e^{a_{\gamma} t} \sum_{y} p_{t}^{\gamma}(x, y) f(y)
$$


here

$$
\left\{\begin{array}{l}
p_{t}^{\gamma}(x, y)=p_{t}^{\gamma}(0, x-y) \text { and } \\
p_{t}^{\gamma}(0, x)=e^{-c^{*} t} \sum_{n=0}^{\infty} \frac{(\beta t)^{n}}{n !} J_{\gamma}^{n}(0, x) \\
\text { with } \\
c^{*}=\beta \hat{J}_{\gamma, 0} \text { and } J_{\gamma}^{n}(0, x)=\sum_{x_{1}, \ldots, x_{n-1}} J_{\gamma}\left(0, x_{1}\right) \ldots J_{\gamma}\left(x_{n-1}, x\right),
\end{array}\right.
$$

i.e. $p_{t}^{\gamma}(x, y)$ is the transition probability of a jump process with intensity $\beta J_{\gamma}(x, y)$. In preparation for the statement of the next lemma and for each $t>0$, we introduce the sets

$$
\mathscr{N}_{t}^{\gamma}=\left\{k \in \mathscr{N}: k \leqq k(\gamma)=s^{-1} \gamma^{-\kappa^{*}} t \lambda^{-2}\right\},
$$

where $\lambda$ is as in the statement of Theorem $4.2, \kappa^{*}>0$ is to be chosen below, $s \in[1,2)$, and

$$
\begin{aligned}
Q_{\gamma, k}= & {\left[-\gamma^{-1-\rho_{1}}-\gamma^{-1}(k(\gamma)-k), \gamma^{-1-\rho_{1}}+\gamma^{-1}(k(\gamma)-k)\right]^{N} \cap \mathbb{Z}^{N} } \\
& \left(k \in \mathscr{N}_{t}^{\gamma} \cup\{0\}\right) ;
\end{aligned}
$$

notice that $Q_{\gamma, k(\gamma)}=Q_{\gamma}$, the latter given by (4.6).

For $k \in \mathscr{N}_{t}^{\gamma}$ let $t_{k}=s \gamma^{\kappa^{*}} k$ and denote by $\sigma^{(k)}$ the spin configuration at time $t=t_{k}$. Then, given any $\zeta>0$, define

$$
\begin{gathered}
H_{t}^{\gamma}=\left\{\sigma^{(0)}:\left\|\sigma^{(k)}-m^{\gamma}\left(\cdot, s \gamma^{\kappa^{*}} \mid \sigma^{(k-1)}\right)\right\|_{\gamma, Q_{\gamma ; k}}<\gamma^{\zeta} \text { for all } k \in \mathcal{N}_{t}^{\gamma}\right\}, \\
H_{0}^{\gamma}=\left\{\sigma^{(0)}:\left\|\sigma^{(0)}-\mathbb{E}_{\mu ;} \sigma^{(0)}\right\|_{\gamma, Q_{; ; 0}}<\gamma^{\zeta}\right\}
\end{gathered}
$$

and

$$
\bar{H}^{\gamma}=H^{\gamma} \cap H_{0}^{\gamma} \text {. }
$$

Lemma 6.3. Assume (6.8). Then for any $T>0, s \in[1,2)$ and all $\zeta<\zeta^{*}=$ $\min \left(\frac{N}{2}, \frac{2 \xi+N}{4}\right)$ and $\xi>\kappa^{*}$, there exist $c_{m}>0$ such that, for all $m \geqq 1$,

$$
\inf _{t \in[0, T]} P_{\mu i}^{\gamma}\left(\bar{H}_{t}^{\gamma}\right) \geqq 1-c_{m} \gamma^{m} .
$$

Proof. 1. It is obvious that it suffices to establish (6.18) for $H_{t}^{\gamma}$ and $H_{0}^{\gamma}$ separately.

2. For $y \in \mathbb{Z}^{N}$ write

$$
p^{\gamma}(y)=p_{\gamma^{\xi}}^{\gamma}(0, y) .
$$

Then

$$
\begin{aligned}
P_{\sigma^{(0)}}^{\gamma}\left(\left(H^{\gamma}\right)^{c}\right) \leqq & S^{-1} \gamma^{-\beta_{*}} t \lambda^{-2}\left|Q_{\gamma, 0}\right| \sup _{\sigma} P_{\sigma}^{\gamma}\left(\mid \sum_{y} p^{\gamma}(y)\left[\sigma^{(1)}(y)\right.\right. \\
& \left.\left.-m^{\gamma}\left(y, t_{1} \mid \sigma^{(0)}\right)\right] \mid>\gamma^{\zeta}\right)
\end{aligned}
$$

where

$$
\left|Q_{\gamma, 0}\right|=\left(\frac{1}{2}\right)^{N}\left(\gamma^{-\rho_{1}-1}+s^{-1} \gamma^{-\kappa^{*}} t \lambda^{-2} \gamma^{-1}\right)^{N}
$$


Using Chebyshev's inequality with power $2 n$ we get

$$
\begin{aligned}
& P_{\sigma}^{\gamma}\left(\sum_{y} p^{\gamma}(y)\left[\sigma^{(1)}(y)-m^{\gamma}\left(y, t_{1} \mid \sigma^{(0)}\right)\right]>\gamma^{\zeta}\right) \\
& \quad \leqq \gamma^{-2 n \zeta} \sum_{y_{1}, \ldots, y_{2 n}}\left[\prod_{i=1}^{2 n} p^{\gamma}\left(y_{i}\right)\right]\left[\mathbb{E}_{\sigma}^{\gamma}\left(\prod_{i=1}^{2 n}\left\{\sigma^{(1)}\left(y_{i}\right)-m^{\gamma}\left(y_{i}, t_{1} \mid \sigma^{(0)}\right)\right\}\right)\right] .
\end{aligned}
$$

3. Lemma 6.2 yields

$$
\sum_{y_{1} \neq \cdots \neq y_{2 n}}\left[\prod_{i=1}^{2 n} p^{\gamma}\left(y_{i}\right)\right]\left[\mathbb{E}_{\sigma}^{\gamma}\left(\prod_{i=1}^{2 n}\left\{\sigma^{(1)}\left(y_{i}\right)-m^{\gamma}\left(y_{l}, t_{1} \mid \sigma^{(0)}\right)\right\}\right)\right] \leqq c_{2 n} \gamma^{N n}
$$

4. The term on the right-hand side of (6.22) with the $y_{i}$ 's pairwise equal is bounded as follows:

$$
\begin{gathered}
\sum_{y_{1} \neq \cdots \neq y_{n}}\left[\prod_{i=1}^{n}\left(p^{\gamma}\left(y_{i}\right)\right)^{2}\right]\left[\mathbb{E}_{\sigma}^{\gamma}\left(\prod_{i=1}^{n}\left\{\sigma^{(1)}\left(y_{i}\right)-m^{\gamma}\left(y_{i}, t_{1} \mid \sigma^{(0)}\right)\right\}\right)^{2}\right] \\
\leqq 2^{2 n} \sum_{y_{1} \neq \cdots \neq y_{n}}\left[\prod_{i=1}^{n}\left(p^{\gamma}\left(y_{i}\right)\right)^{2}\right],
\end{gathered}
$$

where $2^{2 n}$ follows from the obvious fact that

$$
\sup _{\sigma} \sup _{(x, t)}\left[|\sigma(x)|+\left|m^{\gamma}(x, t \mid \sigma)\right|\right] \leqq 2 .
$$

To estimate the right-hand side of (6.24) we write

$$
p^{\gamma}(x)=p_{\gamma^{\xi}}^{\gamma}(0, x)=\mathbf{1}_{0}^{\gamma}(x)+\pi^{\gamma}(x)
$$

where

$$
\pi^{\gamma}(x)=e^{-c^{*} \gamma^{\xi}} \sum_{n=1}^{\infty} \frac{\left(\beta \gamma^{\xi}\right)^{n}}{n !} J_{\gamma}^{n}(0, x) \text { and } \mathbf{1}_{0}^{\gamma}(x)= \begin{cases}e^{-c^{*} \gamma^{\xi}} & \text { if } x=0, \\ 0 & \text { otherwise . }\end{cases}
$$

Using (6.25) and (6.26) and performing the obvious algebraic manipulations we get

$$
\sum_{y_{1} \neq \cdots \neq y_{n}}\left[\prod_{i=1}^{n}\left(p^{\gamma}\left(y_{i}\right)\right)^{2}\right] \leqq \bar{c}_{n}\left[\sum_{y_{i}}\left(\pi^{\gamma}\left(y_{i}\right)\right)^{2}\right]^{n-1}
$$

where $\bar{c}_{n}=O(n)$.

Applying Minkowski's inequality to the right-hand side of (6.27) and using (6.26) we obtain

$$
\sum_{y}\left(\pi^{\gamma}(y)\right)^{2} \leqq c \gamma^{2 \xi+N}
$$

In view of the above, (6.27) becomes

$$
\sum_{y \neq \cdots \neq y_{n}}\left[\prod_{i=1}^{n}\left(p^{\gamma}\left(y_{i}\right)\right)^{2}\right] \leqq c \bar{c}_{n} \gamma^{(2 \xi+N)(n-1)},
$$


hence, going back to (6.24),

$\sum_{y_{1} \neq \sum_{n}}\left[\prod_{i=1}^{n}\left(p^{\gamma}\left(y_{i}\right)\right)^{2}\right]\left[\mathbb{E}_{\sigma}^{\gamma}\left(\prod_{i=1}^{n}\left\{\sigma^{(1)}\left(y_{i}\right)-m^{\gamma}\left(y_{i}, t_{1} \mid \sigma^{(0)}\right)\right\}\right)^{2}\right] \leqq c_{n} \gamma^{(2 \xi+N)(n-1)}$,

where again $c_{n}$ denotes a uniform constant of order $n$.

5. Since all the other terms appearing in the sum on the right-hand side of (6.22) can be bounded by a combination of terms as in (6.23) and (6.28), using (6.20) and (6.21), we obtain

$$
P_{\sigma(0)}^{\gamma}\left(\left(H_{t}^{\gamma}\right)^{c}\right) \leqq c_{n}\left[t^{N}\left(\gamma^{-\kappa^{*}} \gamma^{-1} \lambda^{-2}\right)^{N}+\gamma^{-N\left(\rho_{1}+1\right)}\right] \max \left[\gamma^{N n}, \gamma^{(2 \xi+N)(n-1)}\right] \gamma^{-2 n \zeta} .
$$

By choosing

$$
\zeta<\zeta^{*}=\min \left[\frac{N}{2}, \frac{2 \xi+N}{4}\right]
$$

and taking $n$ large we conclude.

6. The inequality for $H_{0}^{\gamma}$ follows as above, the only difference being that, in view of (4.5) (i), step 4 is trivial.

The next lemma is the basic step in the proof of Theorem 4.2. To state it we need to introduce the solutions $\underline{M}^{\lambda}$ and $\bar{M}_{\lambda}$ of the initial value problems

$$
\left\{\begin{array}{l}
\bar{M}_{t}^{\lambda}+\bar{M}^{\lambda}-\tanh \left[\beta\left(J * \bar{M}^{\lambda}+a \lambda\right)\right]=0 \text { in } \mathbb{R}^{N} \times(0, \infty), \\
\bar{M}^{\lambda}(r, 0)=q\left(\frac{d_{0}(\lambda r)+\varepsilon}{\lambda}, \lambda a\right) \quad\left(r \in \mathbb{R}^{N}\right),
\end{array}\right.
$$

and

$$
\left\{\begin{array}{l}
\underline{M}_{t}^{\lambda}+\underline{M}^{\lambda}-\tanh \left[\beta\left(J * \underline{M}^{\lambda}-a \lambda\right)\right]=0 \text { in } \mathbb{R}^{N} \times(0, \infty), \\
\underline{M}^{\lambda}(r, 0)=q\left(\frac{d_{0}(\lambda r)-\varepsilon}{\lambda},-\lambda a\right) \quad\left(r \in \mathbb{R}^{N}\right)
\end{array}\right.
$$

where $a, \varepsilon>0, d_{0}$ is as in (4.3) and the traveling waves are normalized so that

$$
q(0, a \lambda)=q(0,-a \lambda)=0 .
$$

In view of the asymptotic properties (3.12) of the $q$ 's, for each fixed $a$ and $\varepsilon$, we may choose $\lambda_{0}=\lambda_{0}(a, \varepsilon)$ so that for all $\lambda \leqq \lambda_{0}$,

$$
q\left(\frac{d_{0}-\varepsilon}{\lambda},-\lambda a\right) \leqq q\left(\frac{d_{0}}{\lambda}, 0\right) \leqq q\left(\frac{d_{0}+\varepsilon}{\lambda}, \lambda a\right) \text { on } \mathbb{R}^{N} .
$$

Lemma 6.4. For any $\delta>0$, choose $\xi>\delta, \zeta<\zeta^{*}=\min \left[\frac{N}{2}, \frac{2 \zeta+N}{4}\right]$ and $\kappa^{*}<$ $\min (\zeta, \xi, 1)$ and $\lambda=\lambda(\gamma)$ such that $\gamma^{-\rho^{*}} \lambda(\gamma) \rightarrow+\infty$ as $\gamma \rightarrow 0$, with $\rho^{*}=$ $\min \left[1-\kappa^{*}, \zeta-\kappa^{*}, \xi-\kappa^{*}\right]$. Let $\bar{M}^{\lambda}, \underline{M}^{\lambda}$ be solutions of (6.30) and (6.31) and choose $\lambda$ small enough so that (6.32) holds. Then there is $\gamma_{0}>0$ such that, for all $\gamma \in\left(0, \gamma_{0}\right)$ and $k \in\{1, \ldots, k(\gamma)-1\}$,

$$
\begin{aligned}
& \sup _{\sigma \in \bar{H}^{i}} \sup _{x \in Q_{\gamma, k+1}}\left(m^{\gamma}\left(x, t_{k+1}-t_{k} \mid \sigma^{(k)}\right)-\bar{M}^{\lambda}\left(\gamma x, t_{k+1}\right)\right) \\
& \quad \leqq c e^{(1+\beta)\left(t_{k+1}-t_{h}-\gamma^{\zeta}\right)} \sup _{\sigma \in \bar{H}^{\prime}} \sup _{x \in Q_{\eta, k}}\left(m^{\gamma}\left(x, t_{k}-t_{k-1} \mid \sigma^{(k-1)}\right)-\bar{M}^{\lambda}\left(\gamma x, t_{k}\right)\right)^{+}
\end{aligned}
$$


and

$$
\begin{aligned}
& \sup _{\sigma \in \bar{H}^{\prime}} \sup _{x \in Q_{;, k+1}}\left(\underline{M}^{\lambda}\left(\gamma x, t_{k+1}\right)-m^{\gamma}\left(x, t_{k+1}-t_{k} \mid \sigma^{(k)}\right)\right) \\
& \leqq c e^{(1+\beta)\left(t_{k+1}-t_{k}-\gamma^{\xi}\right)} \sup _{\sigma \in \bar{H}^{\prime}} \sup _{x \in Q_{\gamma, k}}\left(\underline{M}^{\lambda}\left(x, t_{k}-t_{k-1} \mid \sigma^{(k-1)}\right)\right. \\
& \quad-m^{\gamma}\left(x, t_{k}-t_{k-1} \mid \sigma^{(k-1)}\right)^{+} .
\end{aligned}
$$

Proof. 1. We only prove the first inequality, the second follows similarly.

2. Applying Lemma 3.1 on $\mathbb{R}^{N} \times\left[t_{k}+\gamma^{\xi}, t_{k+1}\right]$ we obtain

$$
\begin{aligned}
& m\left(\gamma x, t_{k+1}\right)-\bar{M}^{\lambda}\left(\gamma x, t_{k+1}\right) \leqq c e^{(1+\beta)\left(t_{k+1}-t_{k}-\gamma^{\xi}\right)} \\
& \quad \times\left[\sup _{y \in Q_{\gamma, k}}\left(m\left(\gamma y, \gamma^{\xi} \mid \sigma^{(k)}\right)-\bar{M}^{\lambda}\left(\gamma y, t_{k}+\gamma^{\xi}\right)\right)^{+}+\left(\gamma^{-\rho_{1}}+(k(\gamma)-k)\right.\right. \\
& -\gamma|x|)^{-1 / 2} e^{\left[\gamma^{-\rho_{1}}+(k(\gamma)-k-\gamma|x|)\right]\left[1-\ln \left(\gamma^{-\rho_{1}}+(k(\gamma)-k)-\gamma|x|\right)\right]} \\
& \left.\quad \times \beta\left(t_{k+1}-t_{k}-\gamma^{\xi}\right)^{\left.\gamma^{-\rho_{1}}+(k(\gamma)-k)\right)-\gamma|x|}\right]-C \beta \lambda a\left(t_{k+1}-t_{k}-\gamma^{\xi}\right) .
\end{aligned}
$$

Since $x \in Q_{\gamma, k+1}$, we have

$$
\begin{gathered}
m\left(\gamma x, t_{k+1}\right)-\bar{M}^{\lambda}\left(\gamma x, t_{k+1}\right) \leqq c e^{(1+\beta)\left(t_{k+1}-t_{k}-\gamma^{\xi}\right)} \\
{\left[\sup _{x \in Q_{;, k}}\left(m\left(\gamma x, \gamma^{\xi} \mid \sigma^{(k)}\right)-\bar{M}^{\lambda}\left(\gamma x, t_{k}+\gamma^{\xi}\right)\right)^{+}+\beta\left(t_{k+1}-t_{k}-\gamma^{\xi}\right)\right]} \\
-C \beta \lambda a\left(t_{k+1}-t_{k}-\gamma^{\xi}\right) .
\end{gathered}
$$

3. Employing the variation of constants formula associated to the semigroup $T^{\gamma}$, as well as Lemma 6.1, we get

$$
m\left(\gamma x, \gamma^{\xi} \mid \sigma^{(k)}\right)=\left[T^{\gamma}\left(\gamma^{\xi}\right) \sigma^{(k)}\right](x)+O\left(\gamma^{\xi}+\gamma\right)
$$

and

$$
\bar{M}^{\lambda}\left(\gamma x, t_{k}+\gamma^{\xi}\right)=\left[T^{\gamma}\left(\gamma^{\xi}\right) \bar{M}^{\lambda}\left(\gamma \cdot, t_{k}\right)\right](x)+O\left(\gamma^{\xi}+\gamma\right)
$$

4. Thus

$$
\begin{aligned}
\sup _{x \in Q_{;, k}} & \left(m\left(\gamma x, \gamma^{\xi} \mid \sigma^{(k)}\right)-\bar{M}^{\lambda}\left(\gamma x, t_{k}+\gamma^{\xi}\right)\right)^{+} \\
& \leqq \sup _{x \in Q_{;, k}}\left(T^{\gamma}\left(\gamma^{\xi}\right)\left[\sigma^{(k)}(\cdot)-\bar{M}^{\lambda}\left(\gamma \cdot, t_{k}\right)\right](x)\right)^{+}+O\left(\gamma^{\xi}+\gamma\right) .
\end{aligned}
$$


Furthermore (6.33), (6.36), the definition of $\bar{H}_{t}^{\gamma}$ and Lemma 6.1 yield,

$$
\begin{aligned}
\sup _{x \in Q_{\gamma k+1}} & \left(m^{\gamma}\left(x, t_{k+1}-t_{k} \mid \sigma^{(k)}\right)-\bar{M}^{\lambda}\left(\gamma x, t_{k+1}\right)\right) \\
\leqq & c e^{(1+\beta)\left(t_{k+1}-t_{k}-\gamma^{\xi}\right)}\left[\sup _{x \in Q_{\gamma, k}}\left(m^{\gamma}\left(x, t_{k}-t_{k-1} \mid \sigma^{(k-1)}\right)-\bar{M}^{\lambda}\left(\gamma x, t_{k}\right)\right)^{+}\right. \\
\quad & \left.\quad \beta\left(t_{k+1}-t_{k}-\gamma^{\xi}\right)+O\left(\gamma^{\xi}+\gamma\right)+\gamma^{\zeta}\right]-C \beta \lambda a\left(t_{k+1}-t_{k}-\gamma^{\xi}\right),
\end{aligned}
$$

for all $\sigma \in \bar{H}_{t}^{\gamma}$.

5. The choice of $\lambda$ implies the result for $\gamma$ sufficiently small.

Lemma 6.5. There exists $\gamma_{0}>0$ such that for any $\gamma \in\left(0, \gamma_{0}\right)$ and all $x \in Q_{\gamma, k}, \sigma \in$ $\bar{H}_{t}^{\gamma}$ and $k=1, \ldots, k(\gamma)$,

$$
\bar{M}^{\lambda}\left(\gamma x, t_{k}\right) \geqq m^{\gamma}\left(x, t_{k}-t_{k-1} \mid \sigma^{(k)}\right) \geqq \underline{M}^{\lambda}\left(\gamma x, t_{k}\right) .
$$

Proof. 1. In view of Lemma 6.4, we only need to prove

$$
\bar{M}^{\lambda}\left(\gamma x, t_{1}\right) \geqq m^{\gamma}\left(x, t_{1} \mid \sigma^{(0)}\right) \geqq \underline{M}^{\lambda}\left(\gamma x, t_{1}\right) \text { for } x \in Q_{\gamma, 1} \text {. }
$$

Next we only prove the first inequality, the second following similarly.

2. Arguing as in the proof of Lemma 6.4, using the definition of $\bar{H}_{t}^{\gamma}$ and (6.32), which of course needs $\gamma$ small, we get

$$
\begin{aligned}
\sup _{\bar{H}_{i}, x \in Q_{i ; 1}} & \left(m^{\gamma}\left(x, t_{1} \mid \sigma^{(0)}\right)-\bar{M}^{\lambda}\left(\gamma x, t_{1}\right)\right) \\
\leqq & c e^{(1+\beta)\left(t_{1}-\gamma^{\xi}\right)}\left[\sup _{\bar{H}^{\prime}, x \in Q_{i ; 0}}\left(T^{\gamma}\left(\gamma^{\xi}\right)\left[\sigma^{(0)}-q\left(\frac{d_{0}(\gamma \lambda \cdot)}{\lambda}, 0\right)\right](x)\right)^{+}\right. \\
& \left.\quad+\beta\left(t_{1}-\gamma^{\xi}\right)+O\left(\gamma^{\xi}+\gamma\right)\right]-\beta \lambda a\left(t_{1}-\gamma^{\xi}\right) \\
\leqq & c e^{(1+\beta)\left(t_{1}-\gamma^{\xi}\right)}\left[\gamma^{\xi}+\beta\left(t_{1}-\gamma^{\xi}\right)+O\left(\gamma^{\xi}+\gamma\right)\right]-C \beta \lambda a\left(t_{1}-\gamma^{\xi}\right) .
\end{aligned}
$$

Choosing $\gamma$ sufficiently small we conclude.

We are now in a position to present the proof of Theorem 4.2.

Proof of Theorem 4.2. 1. Let $t>0$ and consider the partition of the interval $\left[0, t \lambda^{-2}\right]$ given by $(6.13)$.

2. Fix $\underline{x} \in \mathbb{Z}_{n}^{N}$. Then

$$
\begin{aligned}
\mathbb{E}_{\mu i}^{\gamma} & {\left[\prod_{x \in \underline{x}} \sigma_{t \lambda^{-2}}(x)-\prod_{x \in \underline{x}} \bar{M}^{\lambda}\left(\gamma x, t \lambda^{-2}\right)\right] } \\
= & \mathbb{E}_{\mu^{\prime}}^{\gamma}\left\{\prod_{x \in \underline{x}} \sigma_{t \lambda-2}(x)-\chi_{\bar{H}_{t}^{\prime}} \mathbb{E}_{\sigma^{(k(\gamma)-1)}}^{\gamma}\left[\prod_{x \in \underline{x}} m^{\gamma}\left(x, s \gamma^{\kappa^{*}} \mid \sigma^{(k(\gamma)-1)}\right)\right]\right. \\
& \left.+\chi_{\bar{H}_{t}^{\prime}} \mathbb{E}_{\sigma^{\left(k\left(\gamma^{\prime}\right)-1\right)}}^{\gamma}\left[\prod_{x \in \underline{x}} m^{\gamma}\left(x, s \gamma^{\kappa^{*}} \mid \sigma^{(k(\gamma)-1)}\right)\right]-\prod_{x \in \underline{x}} \bar{M}^{\lambda}\left(\gamma x, t \lambda^{-2}\right)\right\} \\
= & \mathrm{E}_{\mu^{\prime}}^{\gamma}\left[\left(1-\chi_{\bar{H}_{t}^{\prime}}\right) \prod_{x \in \underline{x}} \sigma_{t \lambda^{-2}}(x)\right]
\end{aligned}
$$




$$
\begin{aligned}
& +\mathbb{E}_{\mu \gamma^{\prime}}^{\gamma}\left\{\chi_{\bar{H}_{t}^{\prime}} \mathbb{E}_{\sigma^{(k(i)-1)}}^{\gamma}\left[\prod_{x \in \underline{x}} \sigma_{t \lambda-2}(x)-\prod_{x \in \underline{x}} m^{\gamma}\left(x, s \gamma^{\kappa^{*}} \mid \sigma^{(k(\gamma)-1)}\right]\right\}\right. \\
& +\mathbb{E}_{\mu^{\prime}}^{\gamma}\left\{\chi_{\bar{H}_{t}^{\prime}} \mathbb{E}_{\sigma^{(k(\gamma)-1)}}^{\gamma}\left[\prod_{x \in \underline{x}} m^{\gamma}\left(x, s \gamma^{\kappa^{*}} \mid \sigma^{(k(\gamma)-1)}\right)-\prod_{x \in \underline{x}} \bar{M}^{\lambda}\left(\gamma x, t \lambda^{-2}\right)\right]\right\} \\
& +\mathbb{E}_{\mu^{\prime}}^{\gamma}\left\{\left(1-\chi_{\bar{H}_{t}^{\prime}}\right) \mathbb{E}_{\sigma^{(k(j)-1)}}^{\gamma} \prod_{x \in \underline{x}} \bar{M}^{\lambda}\left(\gamma x, t \lambda^{-2}\right)\right\} .
\end{aligned}
$$

We denote the terms in the right-hand side of the last equality by (I), (II), (III) and (IV) successively. Below we analyze each one of them separately.

3. For each $n \in \mathbb{N}$, Lemma 6.3 yields a uniform constant $C_{n}$ such

$$
(\mathrm{I})=\mathbb{E}_{\mu \nu}^{\gamma}\left[\left(1-\chi_{\bar{H}_{t}^{\prime}}\right) \prod_{x \in \underline{x}} \sigma_{t \lambda^{-2}}(x)\right] \leqq C_{n} \gamma^{n}
$$

and

$$
(\mathrm{IV})=\mathbb{E}_{\mu \gamma^{\prime}}^{\gamma}\left\{\left(1-\chi_{\bar{H}_{t}^{\prime}}\right) \mathbb{E}_{\sigma^{(k(i)-1)}}^{\gamma} \prod_{x \in \underline{x}} \bar{M}^{\lambda}\left(\gamma x, t \lambda^{-2}\right)\right\} \leqq C_{n} \gamma^{n} .
$$

4. Using Lemma 6.2 we obtain the following, uniform in $\gamma$, bound on (II):

$$
(\mathrm{II})=\mathbb{E}_{\mu^{i}}^{\gamma}\left\{\chi_{\bar{H}_{t}^{\prime}} \mathbb{E}_{\sigma^{(k(\gamma)-1)}}^{\gamma}\left[\prod_{x \in \underline{x}} \sigma_{t \lambda^{-2}}(x)-\prod_{x \in \underline{x}} m^{\gamma}\left(x, s \gamma^{\kappa^{*}} \mid \sigma^{(k(\gamma)-1)}\right]\right\} \leqq 2 n c \gamma^{\frac{N}{2}} .\right.
$$

5. Finally

$$
(\mathrm{III}) \leqq \mathbb{E}_{\mu \gamma}^{\gamma}\left\{\chi_{\bar{H}_{t}^{\prime}} \mathbb{E}_{\sigma^{(k(i)-1)}}\left\{\sum_{i=1}^{n} c_{i}\left|m^{\gamma}\left(x_{i}, s \gamma^{\kappa^{*}} \mid \sigma^{(k(\gamma)-1)}\right)-\bar{M}^{\lambda}\left(\gamma x_{i}, t \lambda^{-2}\right)\right|\right\}\right\},
$$

for some constants $c_{i}$. In addition, Lemmas 3.1 and 6.5 and the fact, in view of (6.32), that

$$
\underline{M}^{\lambda} \leqq \bar{M}^{\lambda} \text { on } \mathbb{R}^{N} \times(0, \infty)
$$

yield

$$
\left|m^{\gamma}\left(x_{i}, s \gamma^{\kappa^{*}} \mid \sigma^{(k(\gamma)-1)}\right)-\bar{M}^{\lambda}\left(\gamma x_{l}, t \lambda^{-2}\right)\right| \leqq \bar{M}^{\lambda}\left(\gamma x_{i}, t \lambda^{-2}\right)-\underline{M}^{\lambda}\left(\gamma x_{i}, t \lambda^{-2}\right) .
$$

Thus

$$
(I I I) \leqq \mathbb{E}_{\mu^{\prime}}^{\gamma}\left\{\chi_{\bar{H}_{t}^{\gamma}} \mathbb{E}_{\sigma^{(k(j)-1)}}^{\gamma}\left\{\sum_{i=1}^{n} c_{i}\left[\bar{M}^{\lambda}\left(\gamma x_{l}, t \lambda^{-2}\right)-\underline{M}^{\lambda}\left(\gamma x_{i}, t \lambda^{-2}\right)\right]\right\}\right\} .
$$

Lemma 3.1 again yields

$$
0 \leqq \bar{M}^{\lambda}\left(\gamma x_{i}, t \lambda^{-2}\right)-\underline{M}^{\lambda}\left(\gamma x_{i}, t \lambda^{-2}\right)=o(1),
$$

as $\gamma \rightarrow 0, \varepsilon \rightarrow 0$ and $a \rightarrow 0$ successively and locally uniformly in $\bigcup_{t>0}\left(P_{t}^{\gamma} \cup N_{t}^{\gamma}\right)$, provided that $|\gamma \lambda x|$ stays bounded. 
6. Summing up the estimates for the terms (I)-(IV), we obtain, for $x \in P_{t}^{r} \cup N_{t}^{r}$

$$
\left|\mathbb{E}_{\mu^{\prime}}^{\gamma}\left[\prod_{x \in \underline{x}} \sigma_{t \lambda^{-2}}(x)-\prod_{x \in \underline{x}} \bar{M}^{\lambda}\left(\gamma x, t \lambda^{-2}\right)\right]\right| \leqq 2\left(C_{n} \gamma^{n}+n c_{1} \gamma^{\frac{N}{2}}\right)+o(1) .
$$

Using Theorem 4.1 we conclude as soon as we estimate the $o(1)$ in (6.38).

4. To this end assume that $(x, t) \in \mathbb{Z}^{N} \times(0, \infty)$ is such that $x \in P_{t}^{\gamma}-$ if $x \in N_{t}^{\gamma}$ we argue similarly - and observe that the proof of Theorem 4.1 yields

$$
\begin{aligned}
\bar{M}^{\lambda}\left(\gamma x, t \lambda^{-2}\right)-\underline{M}^{\lambda}\left(\gamma x, t \lambda^{-2}\right) \leqq & \left(\frac{w_{a}(\gamma \lambda x, t)+o(1)}{\lambda}, \lambda a\right) \\
& -q\left(\frac{w_{-a}(\gamma \lambda x, t)+o(1)}{\lambda},-\lambda a\right),
\end{aligned}
$$

with the $o(1)$ uniform for $|\gamma \lambda x|$ bounded.

An elementary estimate also shows:

$$
w-\delta-c(a) t \leqq w_{-a} \leqq w_{a} \leqq w+\delta+c(a) t \text { on } \mathbb{R}^{N} \times(0, \infty) .
$$

Let us now assume, without any loss of generality, that $\gamma \lambda x \rightarrow r \in \mathbb{R}^{N}$ and that $w(r, t)=\eta>0$. Choosing $\varepsilon, a$ and $\gamma$ sufficiently small and using (3.12) and (3.13) we then have

$$
\begin{aligned}
\bar{M}^{\lambda}\left(\gamma x, t \lambda^{-2}\right)-\underline{M}^{\lambda}\left(\gamma x, t \lambda^{-2}\right) & \leqq m_{\beta, \lambda a}^{+}-q\left(\frac{\eta}{4 \lambda},-\lambda a\right) \leqq m_{\beta, \lambda a}^{+}-m_{\beta}+\frac{a_{2} e}{a_{1}} e^{\frac{a_{1} \eta}{4 \lambda}} \\
& =O(\lambda a)+\frac{a_{2}}{a_{1}} e^{\frac{a_{1} \eta}{4 \lambda}}
\end{aligned}
$$

where the estimate on $m_{\beta, i a}^{+}-m_{\beta}$ is an immediate consequence of the fact that they satisfy (3.3).

We conclude remarking that the dominant term in the error on the right-hand side of (6.38) is of order $\lambda$.

The following is an immediate consequence of the previous proof and Lemma 6.3.

Corollary 6.6. Under the assumptions of Theorem 4.2,

$$
\begin{aligned}
\left.\sup _{\left[\delta \leqq t \leqq t_{*}\right]} P\left(\sup _{x \in Q_{i} \cap\left(P_{t}^{\prime \prime} \cup N_{t}^{*}\right)} T^{\gamma}\left(\gamma^{\xi}\right)\left[\sigma_{t \lambda^{-2}}(x)-\left(\mathbf{1}_{P_{t}}(x) m_{\beta}-\mathbf{1}_{N_{t}}(x) m_{\beta}\right)\right)\right]>\gamma^{\zeta}+\lambda(\gamma)\right) \\
\quad \leqq c_{n} \gamma^{n},
\end{aligned}
$$

for all $n$ and $c_{n}$ as in Lemma 6.3 and $t_{*}$ is the extinction time of $\Gamma_{t}$.

To prove Corollary 6.6 we argue as in the proof of Theorem 4.2. The only difference is that we use $H^{\gamma}$-type sets which are defined for one time step but $k(\gamma)$ times together with a variance of Lemma 6.5 at each iteration. Finally we can get a uniform estimate in time after a careful look at the proof of Lemma 6.3.

(ii) Remarks and generalizations. In the case of non-zero external magnetization field $h$ one obtains different limits. More precisely, if $h=\lambda a$, as $\gamma \rightarrow 0$, a straightforward modification of the proof of Theorem 4.2 will yield a front, at the macroscopic limit, moving with normal velocity

$$
V=\theta \kappa+c(a),
$$

where $c(a)$ as in (3.13). 
If on the other hand, $|h| \geqq h_{0}$ as $\gamma \rightarrow 0$, then we need to use a different scaling, namely $(x, t) \rightarrow(\gamma \lambda x, \lambda t)$, in which case, the resulting front moves with velocity

$$
V=c(n)
$$

i.e. there is not a transport coefficient. The proof of this statement goes along the lines of the proof of Theorem 4.2, hence we choose not to present any details.

Acknowledgements. We would like to thank E. Presutti for making his papers available even at a preliminary form and L.C. Evans for bringing to our attention Jerrard's results.

\section{References}

[AC] Allen, S., Cahn, J.: A microscopic theory for antiphase boundary motion and its application to antiphase domain coarsening. Act. Metall. 27, 1089-1095 (1979)

[B] Bonaventura, L.: Motion by curvature in an interacting spin system. Preprint

[BSS] Barles, G., Soner, H.M., Souganidis, P.E.: Front Propagation and Phase Field Theory. Siam J. Cont. Opt. 31, 439-469 (1993)

[C] Comets, F.: Nucleation for a long range magnetic model. Ann. Inst. H. Poincaré 23, 135-178 (1987)

[CE] Comets, F., Eisele, T.: Asymptotic dynamics, noncritical and critical fluctuations for a geometric long range interacting model. Commun. Math. Phys. 118, 531-568 (1988)

[CGG] Chen, Y.-G., Giga, Y., Goto, S.: Uniqueness and existence of viscosity solutions of generalized mean curvature flow equations. J. Diff. Geom. 33, 749-786 (1991)

[CIL] Crandall, M.G., Ishii, H., Lions, P.L.: User's guide to viscosity solutions of second order partial differential equations. Bul. AMS 27, 1-67 (1992)

[DD] Dal Passo, R., De Mottoni, P.: The heat equation with a non-local density dependent advection term. Preprint

[DGP] De Masi, A., Gobron, T., Presutti, E.: Traveling fronts in nonlocal evolution equations. Preprint

[DOPT1] De Masi, A., Orlandi, E., Presutti, E., Triolo, L.: Glauber evolution with Kač potentials: I. Mesoscopic and macroscopic limits, interface dynamics. Preprint

[DOPT2] De Masi, A., Orlandi, E., Presutti, E., Triolo, L.: Glauber evolution with Kač potentials: II. Spinodal decomposition. Preprint

[DOPT3] De Masi, A., Orlandi, E., Presutti, E., Triolo, L.: Motion by curvature by scaling non local evolution equations. Preprint

[DOPT4] De Masi, A., Orlandi, E., Presutti, E., Triolo, L.: Stability of the interface in a model of phase separation. Proc. Royal Soc. Edinb., to appear

[DP] De Masi, A., Presutti, E.: Mathematical Methods for Hydrodynamic Limits. Lecture Notes in Mathematics, Berlin, Heidelberg, New York: Springer, 1991

[E] Evans, L.C.: The perturbed test function method for viscosity solutions of non-linear PDE. Proc. Royal Soc. Edinb. 111A, 359-375 (1989)

[ES] Evans, L.C., Spruck, J.: Motion of level sets by mean curvature I.J. Diff. Geom. 33, 635-681 (1991)

[ESS] Evans, L.C., Soner, H.M., Souganidis, P.E.: Phase transitions and generalized motion by mean curvature. Comm. Pure Appl. Math. XLV, 1097-1123 (1992)

[G] Gurtin, M.E.: Multiphase thermomechanics with interfacial structure. 1. Heat conduction and the capillarity balance law. Arch. Rat. Mech. Anal. 104, 185-221 (1988)

[HL] Hemmer, P.C., Lebowitz, J.L.: Systems with weak long-range potentials. In: Phase transitions and critical phenomena, Vol 5b, Eds Domb, C., Green, M.S., London: Academic Press, 1976

[I] Ilmanen, T.: Convergence of the Allen-Cahn equation to Brakke's motion by mean curvature. J. Diff. Geom. 38, 417-461 (1993)

[IS] Ishii, H., Souganidis, P.E.: Generalized motion of noncompact hypersurfaces with velocity having arbitrary growth on the curvature tensor. Tohuko Math. J., in Press 
[J] Jerrard, R.: Fully nonlinear phase field equations and generalized mean curvature motion. CPDE to appear

[KS] Katsoulakis, M.A., Souganidis, P.E.: Interacting particle systems and generalized mean curvature evolution. Arch. Rat. Mech. Anal., to appear

[KUH] Kac, M., Uhlenbeck, G.E., Hemmer, P.C.: On the Van der Waals theory of vapor-liquid equilibrium. I. Discussion of an one-dimensional model. J. Math. Phys. 4, 216-228 (1963)

[L] Liggett, T.: Interacting particle systems. Berlin, Heidelberg, New York: Springer, 1985

[LOP] Lebowitz, J.L., Orlandi E., Presutti, E.: A particle model for spinodal decomposition. J. Stat. Phys. 63, 933-975 (1991)

[LP] Lebowitz, J., Penrose, O.: Rigorous treatment of the Van der Waals Maxwell theory of the liquid vapour transition. J. Math. Phys. 98, 98-113 (1966)

[LPV] Lions, P.L., Papanicolaou, G., Varadhan, S.R.S.: Homogenization of Hamilton-Jacobi equations. Preprint

[P] Penrose, O.: A mean field equation of motion for the dynamic Ising model. J. Stat. Phys. 63, 975-986 (1991)

[So1] Soner, H.M.: Motion of a set by the curvature of its boundary. J. Diff. Eq. 101, 313-372 (1993)

[So2] Soner, H.M.: Ginzburg-Landau equation and motion by mean curvature, I: Convergence. J. Geom. Anal., to appear

[Sp] Spohn, H.: Interface motion in models with stochastic dynamics. J. Stat. Phys. 71, 10811132 (1993)

Communicated by T. Spencer 
